Article

\title{
Ramjet Nozzle Analysis for Transport Aircraft Configuration for Sustained Hypersonic Flight
}

\author{
Raman Baidya ${ }^{1}$, Apostolos Pesyridis ${ }^{1,2, *}$ and Maxim Cooper ${ }^{1}$ \\ 1 College of Engineering and Design, Brunel University London, Uxbridge UB8 3PH, London, UK; \\ me12rrb@my.brunel.ac.uk (R.B.); 1313111@brunel.ac.uk (M.C.) \\ 2 Metapulsion Engineering Ltd., Northwood HA6 3LG, London, UK \\ * Correspondence: a.pesyridis@metapulsion.com; Tel.: +44-1895-267-901
}

Received: 5 March 2018; Accepted: 4 April 2018; Published: 6 April 2018

\begin{abstract}
For the past several decades, research dealing with hypersonic flight regimes has been restricted mainly to military applications. Hypersonic transportation could be a possible and affordable solution to travel in the medium term and there is renewed interest from several private organisations for commercial exploitation in this direction. Various combined cycle propulsion configurations have been proposed and the present paper deals with implications for the nozzle component of a ramjet configuration as part of one such combined cycle propulsion configuration. An investigation was undertaken for a method of turbine-based propulsion which enables the hypersonic vehicle to take off under its own power and propel the aircraft under different mission profiles into ramjet operational Mach regimes. The present study details an optimal method of ramjet exhaust expansion to produce sufficient thrust to propel the vehicle into altitudes and Mach regimes where scramjet operation can be initiated. This aspect includes a Computational Fluid Dynamics (CFD)-based geometric study to determine the optimal configuration to provide the best thrust values. The CFD parametric analysis investigated three candidate nozzles and indicated that the dual bell nozzle design produced the highest thrust values when compared to other nozzle geometries. The altitude adaptation study also validated the effectiveness of the nozzle thrust at various altitudes without compromising its thrust-producing capabilities. Computational data were validated against published experimental data, which indicated that the computed values correlated well with the experimental data.
\end{abstract}

Keywords: hypersonic; propulsion; ramjet; nozzle; convergent-divergent; expansion; shock wave

\section{Introduction}

Hypersonic travel in recent decades has been an important area of research interest in the aerospace sector due to its potential in military and civil applications. In particular, hypersonic air-breathing propulsion vehicles can offer a replacement for the conventional subsonic modes of commercial transport with a new class of flight vehicles that can offer time-saving means of transportation for long-range flights.

Historically, the approach to hypersonic flight research has spanned several decades with constant improvements in performance through improvements in aerodynamics, structures, and propulsion systems leading to successively higher flight speeds. For flight above Mach 3, the main representative was the Lockheed SR-71, which utilised Turbine-Based Combined Cycle (TBCC) engines to propel the aircraft. Powered by two Pratt and Whitney J-58 engines, the aircraft was capable of achieving Mach 3.5 flight speeds, making it the fastest air-breathing aircraft to take off under its own power [1].

Perhaps the most noteworthy aeronautical research effort in history is the rocket-powered hypersonic research aircraft X-15 that holds the record for the fastest manned aircraft achieving a speed 
of Mach 6.72. The structure of the aircraft was influenced by intercontinental missile development research being conducted at the time, and it was capable of reaching an altitude of over 350,000 feet, demonstrating that the pilots can fly rocket-powered vehicles from the Earth's atmosphere to the edge of outer space and returning with valuable data utilised in the design of succeeding aircrafts and spacecrafts [2]. Since the termination of the X-15 project in 1968, studies of hypersonic flight have been largely limited to missiles and research aircrafts owing to the complications arising from structural requirements, unsatisfactory fuel economy, and recurrent maintenance issues as well as specific propulsion system challenges of air-breathing engines in these flight regimes.

\subsection{High-Speed Commercial Transportation}

The epitome of successful high-speed (i.e., supersonic) commercial transportation aircraft was the Concorde, a joint collaboration project between Britain and France which revolutionised commercial air travel by cutting down air journey time by half-bridging London and New York with only a 3.5-h flight time. At the same time efforts in the then Soviet Union resulted in the Tu-144 aircraft which was, however, less successful and short-lived. The Concorde was capable of Mach 2 cruise speeds, powered by four Rolls-Royce/Snecma Olympus 593 afterburning turbojets capable of producing a maximum installed thrust of 152,200 lbs [3].

However, the significant fuel consumption coupled with escalating fuel prices and fears of another oil crisis caused its retirement as its popularity plummeted.

Presently, there exists a large gap in the market, primarily business travellers, for the potential revival of supersonic, or better yet hypersonic travel. Focussing available resources on the design and development of airliners capable of achieving hypersonic cruise speeds can be achieved by extending the research on air breathers.

\subsection{Hypersonic Propulsion}

Hypersonic air-breathing vehicles utilise the air they operate in to propel the vehicle to speeds ranges beyond the Mach 5 ( 5 times the local speed of sound) envelope. Historically, rocket-based propulsive systems have been utilised for the purpose of propelling aircrafts and spacecrafts to hypersonic flight speeds. However, air-breathing modes of propulsion tend to be preferable for sustained atmospheric flights due to their virtue of operation without the necessity of carrying oxidisers, resulting in significant weight and space savings and thus allowing for a proportionately much larger payload to be carried.

Air-breathing engines for hypersonic vehicles require a scramjet system to operate and are often proposed in combined cycle form in that they also contain a conventional gas turbine and ramjet in the same package. The reason for this is that each engine can operate within a different flight speed regime and all three are required for successful operation at hypersonic speeds.

The present paper focusses on the intermediate component: the ramjet. Engines that take advantage of ram air compression are classified as ramjet engines, which eliminate the need to rely on axial compression. The compression is achieved in the aerodynamic diffusing (ram) process created by the inlet and diffuser. The engine utilises the aircraft's forward momentum for the compression of air, requiring the ramjet engine to operate supersonically. This renders the engine practically inoperable at lower Mach numbers.

Figure 1 depicts the schematic of an ideal ramjet. The engine consists of a diffuser, a combustion chamber, and an exhaust nozzle. A typical ramjet includes an air inlet followed by a diffuser, allowing the air to be supersonically decelerated to subsonic conditions and compressed utilising a train of shocks, accompanying a rise in temperature that provides ample conditions for the air being fed into the combustion chamber [4]. 


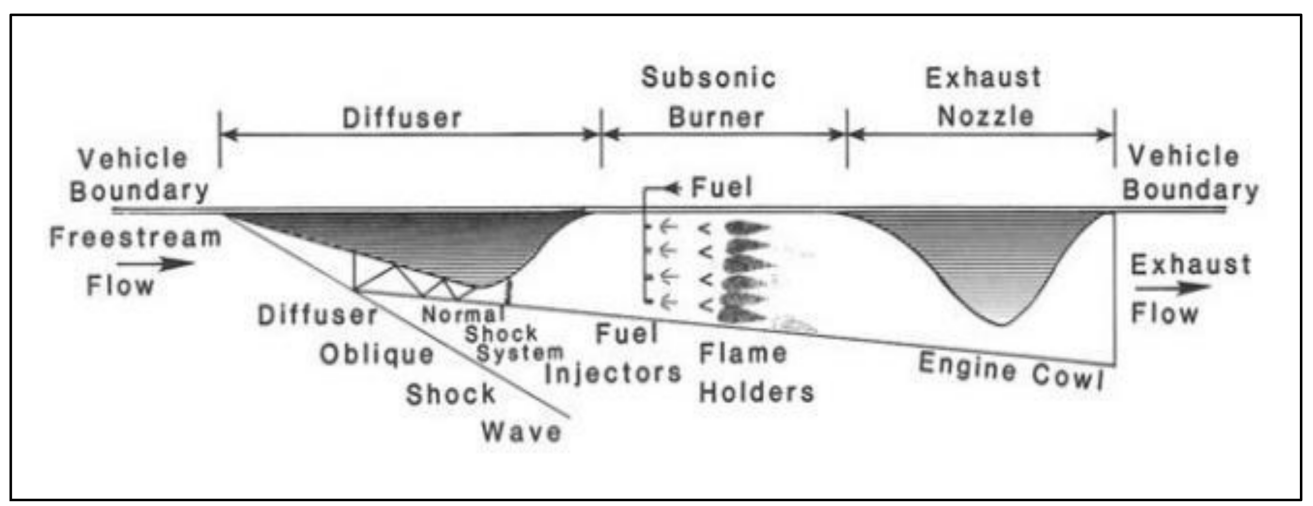

Figure 1. Ramjet engine schematic.

The compressed air is mixed with fuel and combusted and fed straight into the exhaust system for expansion, contrary to gas-turbine engines, where high temperature gas is passed through the turbines utilised to drive the compressors. The nozzle, of convergent-divergent geometry, accelerates the high temperature gas from subsonic at the nozzle inlet to supersonic speeds, creating a high velocity exit jet producing the required positive momentum for thrust generation from the overall engine. Ramjets are typically utilised primarily for military applications for the propulsion of missiles. Nevertherless, aircrafts have also utilised ramjets for propulsion. Examples include the SR-71 Blackbird, which featured a turboramjet propulsion system capable of Mach 3.5 speeds.

In the present paper, a concept aircraft was powered by a combined cycle hypersonic propulsion system which features a gas turbine, ramjet, and scramjet layout, and was first presented in Reference [5]. The focus was on maximising the thrust component from the ramjet as an important criterion and pre-cursor to allowing the aircraft to achieve hypersonic speeds.

To achieve the above goal, three different nozzle types were investigated. Namely, conical, bell, and dual bell nozzles were tested to comprehensively investigate the possibilities available to the propulsion system designer with respect to nozzle type choices and their specific features. This paper focussed on axisymmetric nozzle configurations without consideration for planar or other three-dimensional nozzle configurations. For this reason, wide-ranging computational fluid dynamics (CFD) was undertaken to highlight the most promising design through a parametric analysis. A full description of the CFD setup and validation is provided in later sections, covering thrust and altitude suitability, while the fundamentals are covered in the sections immediately following.

\section{Concept Aircraft Specification}

The hypersonic combined cycle propulsion concept is based around a self-sustained concept aircraft required to achieve a full commercial-style flight profile for passenger transportation. An example outline of the desired profile is given in Table 1. In order to remain self-sustained, the profile is attained by the combined operation of a turbojet, ramjet, and scramjet propulsion system.

A feasible combination of a dual-mode ramjet-scramjet is given by Figure 2, such that during high-altitude scramjet flight the ramjet inlets are sealed to prevent excessive drag induced by shockwaves on the ramjet forebody [5].

Table 1. Concept aircraft target flight profile.

\begin{tabular}{|c|c|c|c|c|c|c|}
\hline Phase & Description & Altitude (ft) & Mach & Turbojet & Ramjet & Scramjet \\
\hline 1 & Take-off and Initial Climb & $0-1500$ & 0 to 1 & & & \\
\hline 2 & Climb 1 & $1500-40,000$ & 1 to 2 & & & \\
\hline 3 & Climb 2 & $40,000-60,000$ & 2 to 4 & & & \\
\hline 4 & Climb 3 & $60,000-100,000$ & 4 to 7 & & & \\
\hline \multirow[t]{2}{*}{5} & Cruise & 100,000 & 8 & & & \\
\hline & Single Operation & & Dual Operation & & & ration \\
\hline
\end{tabular}




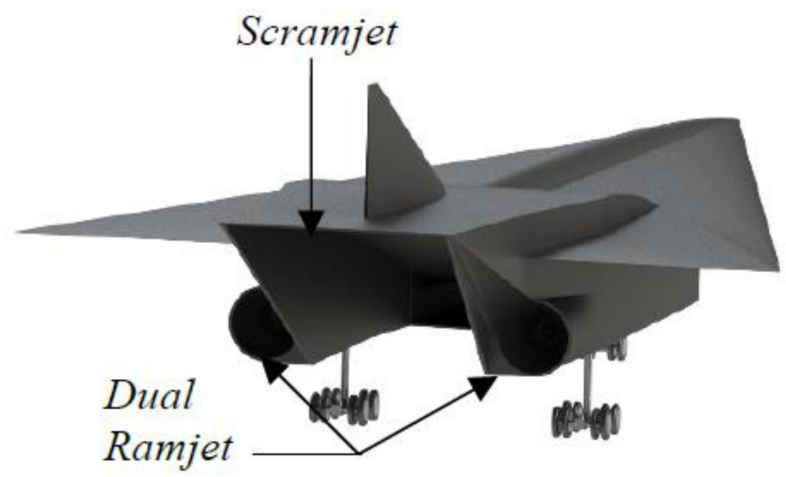

Figure 2. Concept hypersonic aircraft.

The requirement from this aircraft was for sustained Mach 8 cruise flight for a substantial part of the flight envelope with the ramjets assisting during phases 2 and 3 of the flight envelope (Table 1). The concept paper as well as it combustion characteristics have been detailed in a previous publication [5].

\section{Conceptual Design of a Ramjet Nozzle}

This section provides a comprehensive discussion of the operating principles of the ramjet component followed by a detailed study of the design requirement of the convergent-divergent nozzle.

Firstly, the ramjet accomplishes compression by decelerating the oncoming airflow to low subsonic Mach numbers such that combustion can stably occur. The shocks are designed to be positioned in the intake system of the ramjet and the oncoming flow is decelerated from supersonic speeds through the shocks $(2<\mathrm{M}<5)$ and compressed. The compression takes place over several steps which include the airflow passing through one or more oblique shock waves generated by the fore body of the vehicle, finally ending with a normal shock after which the flow becomes subsonic. Then, subsonic diffusion takes place through a normal diffusion process. The process of supersonic compression followed by subsonic diffusion converts a major proportion of the kinetic energy of the oncoming flow to static pressure, which is fed into the combustion chamber (Figure 3) through stations 0 (free stream), 1 (inlet), 2 (combustor inlet), 3 (nozzle inlet), and 4 (nozzle exit).

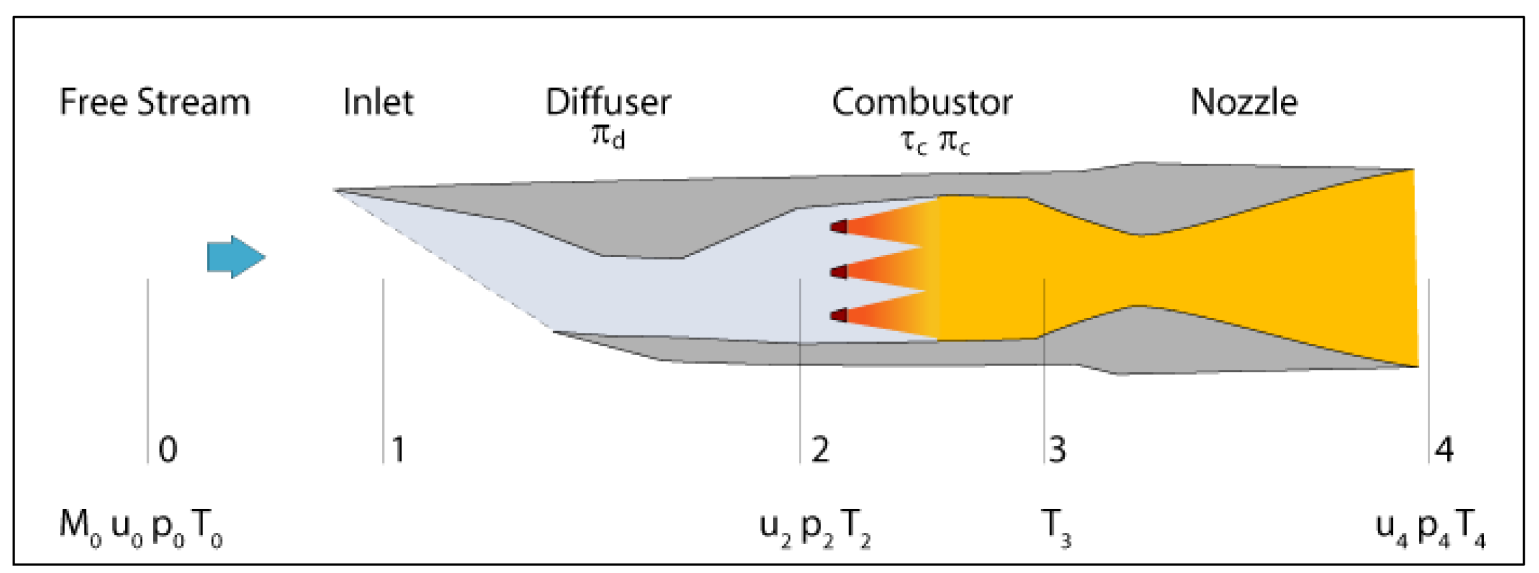

Figure 3. Thermodynamic stations of a ramjet.

Succeeding the compression process, the flame holders process the normal combustion phenomenon, similar to conventional gas turbine engines. The lower, subsonic speeds lead to efficient combustion, after which the flow is allowed to mix up to allow a uniform flow profile of temperature and pressure which is fed into the convergent-divergent nozzle. This nozzle accelerates the high temperature subsonic gas into the atmosphere, essentially creating an exit jet and producing thrust. 
The following section elaborates on the design of the propelling nozzle for a ramjet engine for larger thrust values required, as well as the effect the geometrical configurations have on the thrust values accompanying any other factors affecting the efficient functioning of the nozzle.

\subsection{Flow through a Convergent-Divergent (C-D) Nozzle}

For C-D nozzles, as the ambient pressure is gradually reduced compared to a reference reservoir pressure. Meanwhile, the flow in the convergent section increases until sonic velocity, which is achieved at the throat (minimum area section). Further reduction in the ambient pressure does not result in an increase in flow velocity. As the flow proceeds past the throat into the divergent section, however, it accelerates further to supersonic speed as depicted in Figure 4a [6], with nozzle nomenclature provided in Figure $4 \mathrm{~b}$ [7]. The pressure differential between the exit pressure $\left(\mathrm{P}_{\mathrm{e}}\right)$, referred to as back pressure, and the reservoir pressure leads to the flow achieving supersonic speeds through the nozzle [8].

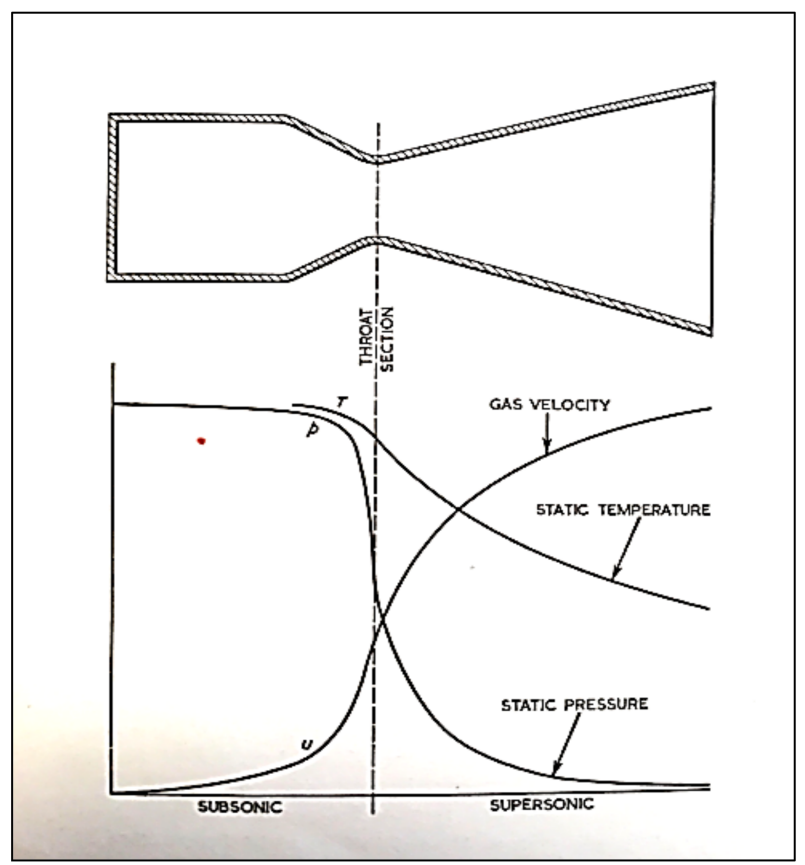

(a)

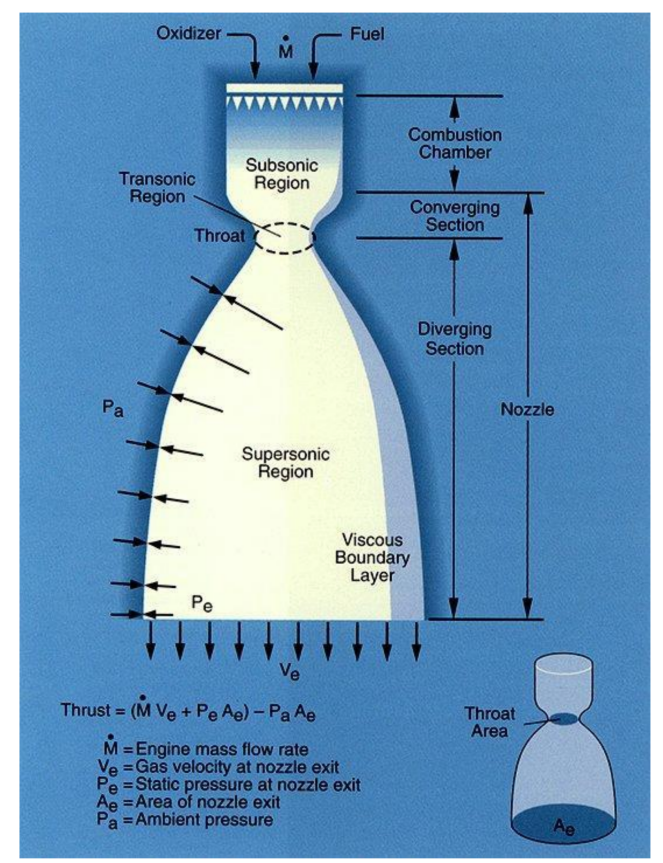

(b)

Figure 4. (a) Expansion in a convergent-divergent (C-D) supersonic nozzle and (b) nozzle geometrical designations.

Most of the gross nozzle trust, produced from jet momentum with additional thrust from pressure, will cause an imbalance between the throat static pressure and ambient pressure. In contrast to convergent nozzles, at high Nozzle Pressure Ratios $(N P R=p t / p 0)$, the C-D nozzle allows expansion to take place inside the nozzle to maximise the thrust being produced. The area ratio between the throat $\left(A^{*}\right)$ and the exit area $\left(A_{e}\right)$ impacts the flow significantly. Downstream of the throat, the nozzle gradually increases in the cross-sectional area, creating the characteristic parabolic bell profile, which allows the exhausted gases to expand, creating the required thrust. They can be characterised by the expansion area ratio $(\varepsilon)$ where:

$$
\varepsilon=\frac{A_{e}}{A^{*}}
$$

\subsection{Characteristics of Flow through Ramjet Nozzles}

In order to design a ramjet nozzle, careful attention needs to be paid to various geometric, structural, and aerodynamics aspects, the complexity of which is multiplied as a result of the highly 
dynamic mission profile. If the ramjet were to operate in the same ambient conditions and environment, it would be sensible to choose one optimum geometric nozzle design would suffice for maximum performance. However, in reality the final design has to compromise within the band of variation created by the change in flight profile and flight speeds associated with ramjet operation. The following section discusses the flow characteristics and associated challenges these impose on ramjet design and the potential nozzle design solution that can be adopted to overcome them.

\subsubsection{Shock Diamond}

Aerospace propulsive systems operating at supersonic Mach numbers including ramjets feature complex aerodynamic phenomena. A key such feature is the creation of shock diamonds, also known as Mach diamonds or disks, which are repeated shock patterns trailing the supersonic nozzle. This condition is caused due to the difference in pressure between the exit pressure $\left(\mathrm{P}_{\mathrm{e}}\right)$ and ambient pressure $\left(\mathrm{P}_{0}\right.$. $)$ Under these conditions, the nozzle loses its efficiency during the thrust generation process. The pressure differential condition can result in either under-expansion $\left(\mathrm{P}_{\mathrm{e}}>\mathrm{P}_{0}\right)$ or over-expansion $\left(\mathrm{P}_{\mathrm{e}}<\mathrm{P}_{0}\right)$. The repeated formations result in friction between the exhausted jet air and the free stream, resulting in a turbulent shear layer which causes a viscous damping effect. This gradually dissipates the wave structure downstream of the nozzle exit [6].

\subsubsection{Under- and Over-Expansion}

As discussed previously, in ideal conditions the nozzle produces adequate thrust under correct expansions conditions. Under non-ideal conditions, the reservoir pressure, i.e., the pressure leaving the combustion chamber according to the geometry of the nozzle, determines the expansion criteria of the gases being exhausted. In cases where the ambient pressure is not equal to the pressure of the supersonic gas stream at the nozzle exit, the gas tries to adjust the conditions prior to or beyond the nozzle exit, thus affecting the thrust levels available.

Under conditions where the ambient pressure is significantly lower than the exit pressure, the expansion continues after leaving the nozzle and deviates from the nozzle axis after suffering what is known as "Prandtl-Meyer Expansion" [6], as depicted in Figure 5a. This leads to a vacuum forming at the axis of the jet, which sucks back the momentum of the gases towards it, creating recompression as the expansion waves are reflected from the constant pressure jet boundary as compression waves. The expansion is produced by wavelets, as depicted in Figure $5 a$, at the opposite jet boundary which, along with the radial expansion, cause a series of small returning shocks which coalesce near the axis to form diamond patterns [6]. The gases therefore return to the conditions at the nozzle exit and the process is repeated, resulting in a phenomenon known as "under-expansion". Since the flow is supersonic, the expansion followed by the compression of the exhaust jet beyond the nozzle is achieved by Mach and shock waves [9].

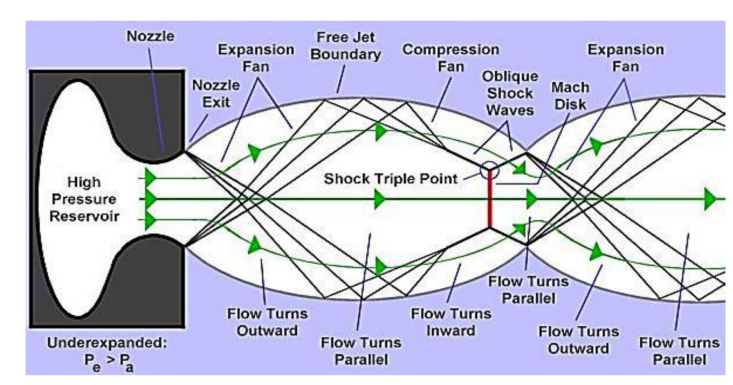

(a)

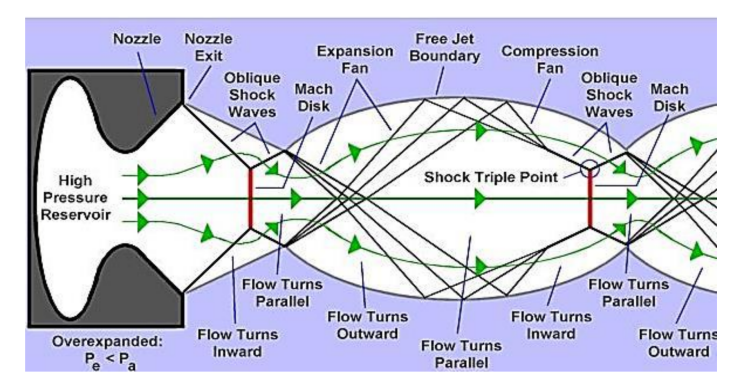

(b)

Figure 5. Shock diamond patterns of (a) under-expansion; (b) over-expansion.

On the other hand, if the outside pressure is higher than that of the nozzle exit, an oblique shock is generated, which raises its pressure and is compressed inwards. However, the compressed flow 
pressure may exceed the atmospheric pressure, in which case the flow expands back outward to reduce the pressure again. This process may be repeated several times until the exhaust pressure is matched with the ambient pressure. In other words, the flow will repeatedly compress and expand while gradually dissipating towards the equalisation of the pressure difference between the atmosphere and the exhaust. A shock diamond pattern is created, which is in this case known as over-expansion (Figure 5b).

\subsection{Nozzle Geometric Configurations}

Historically, the shape of the diverging section of the nozzle has been primarily conical owing to the simplicity and the ease of construction. However, in time, a better understating of the aerothermodynamics of convergent-divergent nozzles has led to the substitution of the conventional conical nozzle with more sophisticated shapes in order to minimise fluid dynamic losses. In addition to the conical nozzle configuration, the bell and dual bell nozzle shapes are described in the following sections owing to their suggested superior performance. The conical nozzle was studied as a reference point for the later comparison of the other two more advanced types.

\subsubsection{Conical Nozzle}

Conical nozzles work on the principle of converting the exhausted gases into axial thrust, and perform best when the axial thrust is uniform throughout the nozzle axis [8]. Hence, smaller divergence angles $(\alpha)$ produce greater axial thrust because they maximise the axial component of the exhaust exit velocity, producing high specific impulse in the process (Figure 6a). However, this does result in longer and heavier nozzle divergent sections, which leads to problems with installation. Shortening the nozzles with large divergence angles leads to divergence losses. In a conical nozzle with a semi-angle $(\alpha)$ divergent nozzle, by the time the exhaust has travelled past the exit, the streamlines have diverged away from the axis of the jet, producing radial thrust instead [7]. Larger divergence angles reduce low altitude performance as high pressures lead to over-expansion, which in turn may lead to flow separation. A divergence loss factor $(\lambda)$ can be given (Equation (2)) to characterise this phenomenon (Figure 6b):

$$
\lambda=\frac{(1+\cos \alpha)}{2}
$$

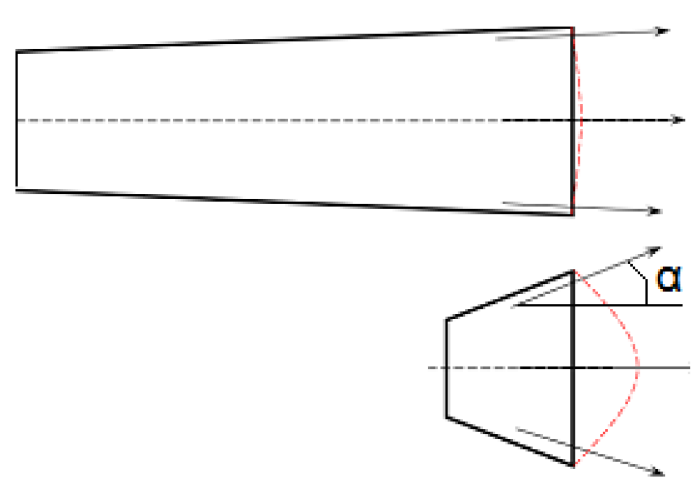

(a)

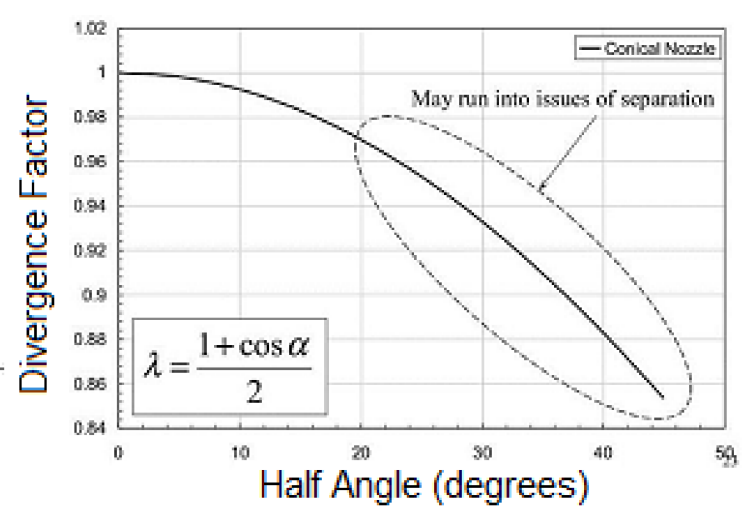

(b)

Figure 6. (a) (Top) Mostly axial thrust, (Bottom) mostly radial thrust; (b) and the divergence factor in relation to the nozzle geometry [8].

\subsubsection{Bell Nozzle}

Bell-shaped nozzles are currently the most commonly utilised nozzle profile. Nozzle profiling in this case is tailored to yield certain desired pressure gradients to minimise flow separation, thus improving the performance of the simple cone nozzle. 
Bell-shaped nozzles consist of two sections. The nozzle contours diverge at a relatively large angle near the throat and taper off further downstream. At the section near the nozzle exit the divergence angle is very small, almost parallel to the jet axis. This configuration allows a maximisation of the performance while minimising the weight, with the length of the nozzle being as much as $25 \%$ lower than the conical nozzle equivalent. This type of nozzle achieves the reduction of divergence by turning the flow to provide an axial flow [8]. Bell nozzles typically exhibit one design point where they are at their most efficient at a given altitude of operation.

In addition, the bell nozzle, similarly to the conical nozzle, exhibits over- and under-expansion flow effects under different altitude and flow conditions. The principal regimes of over- and under-expansion for both nozzles are presented in Figure 7 below.

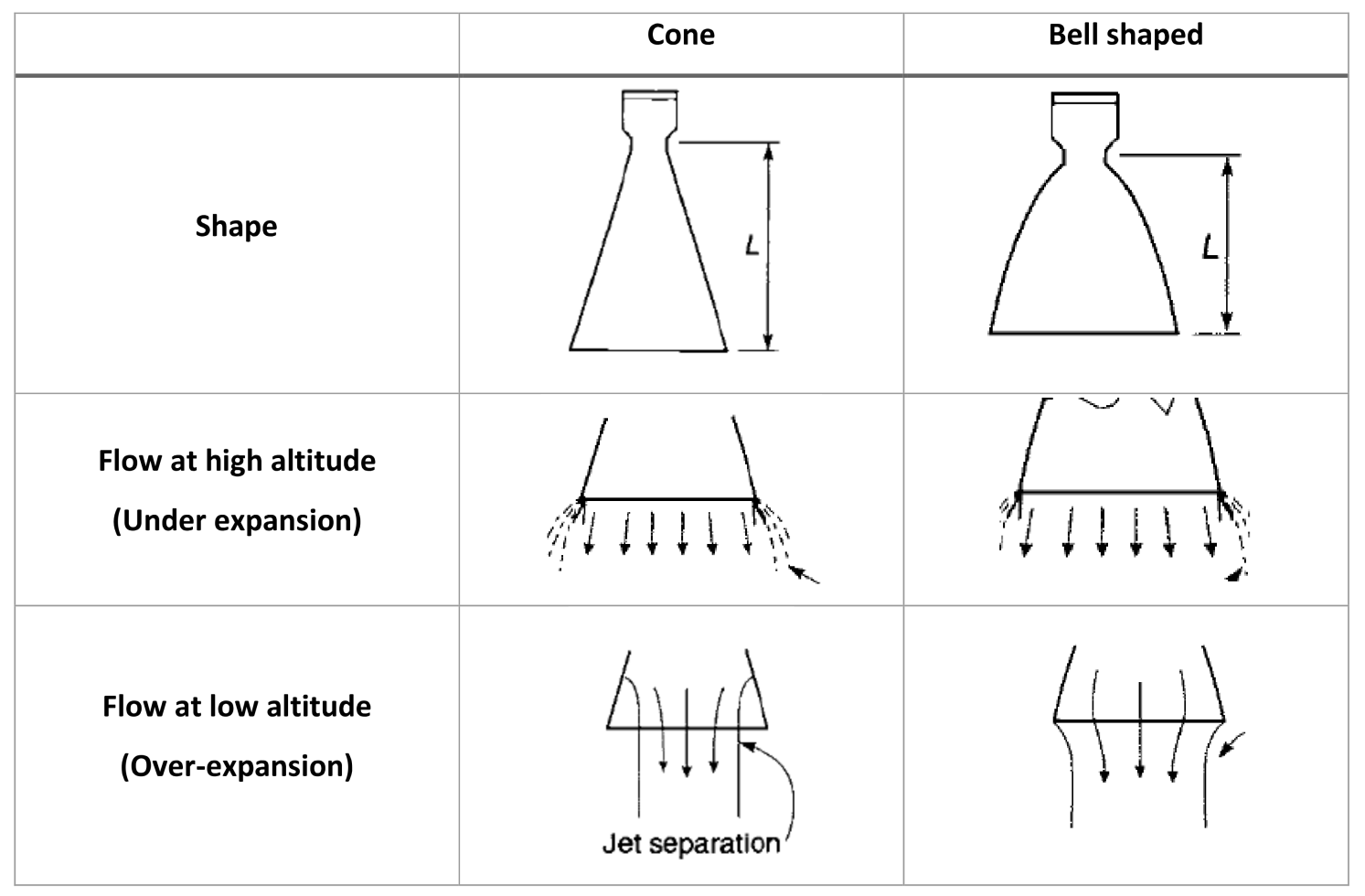

Figure 7. Nozzle performance at different altitudes.

The bell nozzle basic geometry is provided in Figure 8, with $\alpha$ being the initial nozzle angle immediately downstream of the throat and $\theta_{e x}$ the final nozzle exit angle.

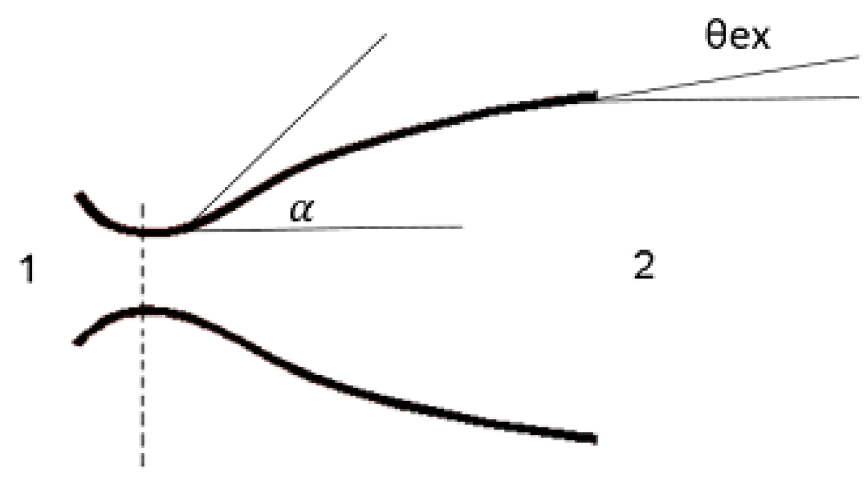

Figure 8. Bell nozzle divergence. 
The divergence loss is characterised by the divergence loss factor, $\lambda$, in Equation (3), below:

$$
\lambda=\frac{1}{2}\left(1+\cos \frac{\left(\alpha+\theta_{e x}\right)}{2}\right)
$$

Truncated idealised contoured (TIC) nozzles (Figure 9, left) are a class of bell-shaped nozzle developed to produce a virtually unidirectional exhaust profile. The walls of the contour curve near the throat region and become nearly parallel to the nozzle axis at the exit plane. However, the nozzle length ought to be equal to 50 times the throat radius in theory. In cases where this requirement cannot be met, for shorter nozzles, the flow is forced to straighten out earlier, causing a decrease in the nozzle efficiency [10].

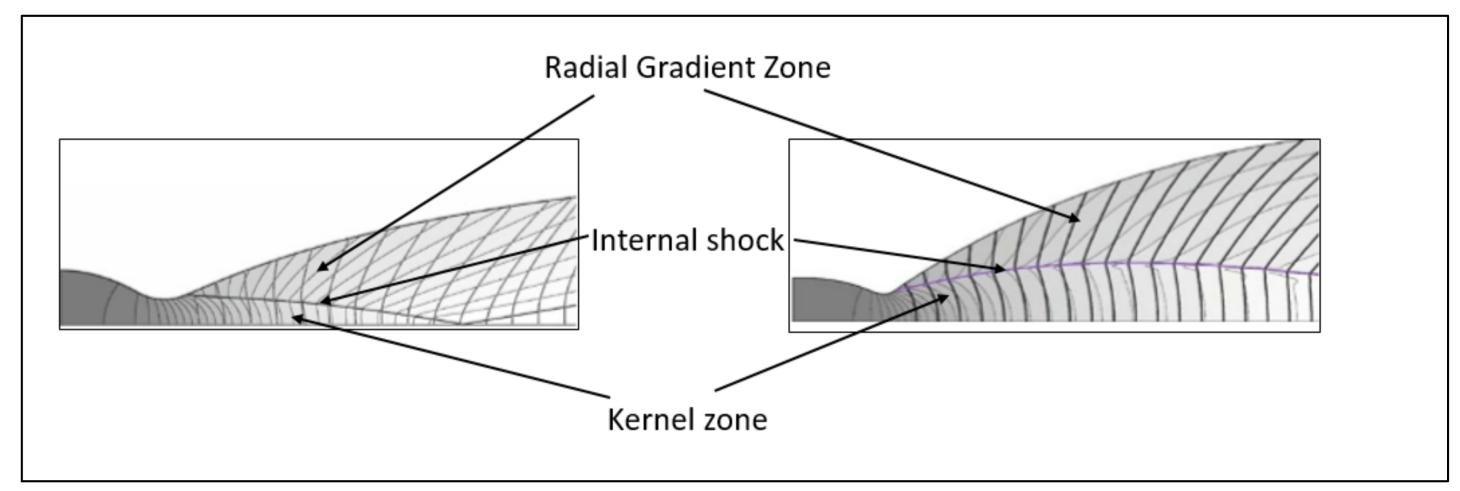

Figure 9. (left) Truncated idealised contoured (TIC) nozzle (right) and thrust-optimised contoured (TOC) nozzle.

Thrust-optimised contoured nozzles (TOC, Figure 9, right) are a step further from TIC nozzles where the near throat region has a shaped gradient of curve in the near throat region which corresponds to the greater expansion of the flow. The higher increase in the parabolic arc induces an internal shock that increases the pressure at the exit plane of the nozzle [10,11].

Bell nozzles can be characterised by the presence of weak compression waves, which lead to the formation of an internal shock induced by the sharp turn in the nozzle succeeding the throat area. The shock separates two regions of varying flow velocities, the kernel region and the radial gradient region. The kernel region is where the flow field depends on the expansion at the throat, involving expansion waves generated at the convex wall of the throat region and its reflection at the throat axis. The larger kernel regions lead to higher jet velocities at the nozzle exit. Radial gradient zones lie between the kernel zone and the wall of the nozzle where the radial pressure gradient is positive, i.e., the pressure increases moving away from the axis. Larger radial gradient zones, such as those in TIC nozzles, lead to separation within the nozzle wall boundaries and result in separation shocks. TOC nozzles, however, have smaller radial gradient zones and larger kernel zones, leading to higher flow velocities at the nozzle exit with a lower possibility of separation.

\subsubsection{Dual Bell and Contoured Nozzle}

The concept of the dual bell nozzle (patented in 1968 by Rocketdyne) offers a solution to the altitude expansion difficulties faced by conventional bell-shaped nozzles. The contour inflection ensures a controlled and symmetrical separation, therefore limiting the generation of high side loads of separated flow in conventional nozzles and increasing thrust (Figure 10, left, top). The area ratio of the smaller nozzle is comparatively much smaller than the reference main engine nozzle, resulting in a better thrust performance at lower altitudes. When ascending to higher altitudes, the separation point leaves the inflection point and moves down the exit plane where a higher area ratio of the nozzle extension provides thrust with minimal losses caused due to the change in altitude pressure (Figure 10, left, top). Dual bell nozzles are also classified as altitude compensating nozzles (ACN) (Figure 10, right) $[11,12]$. 

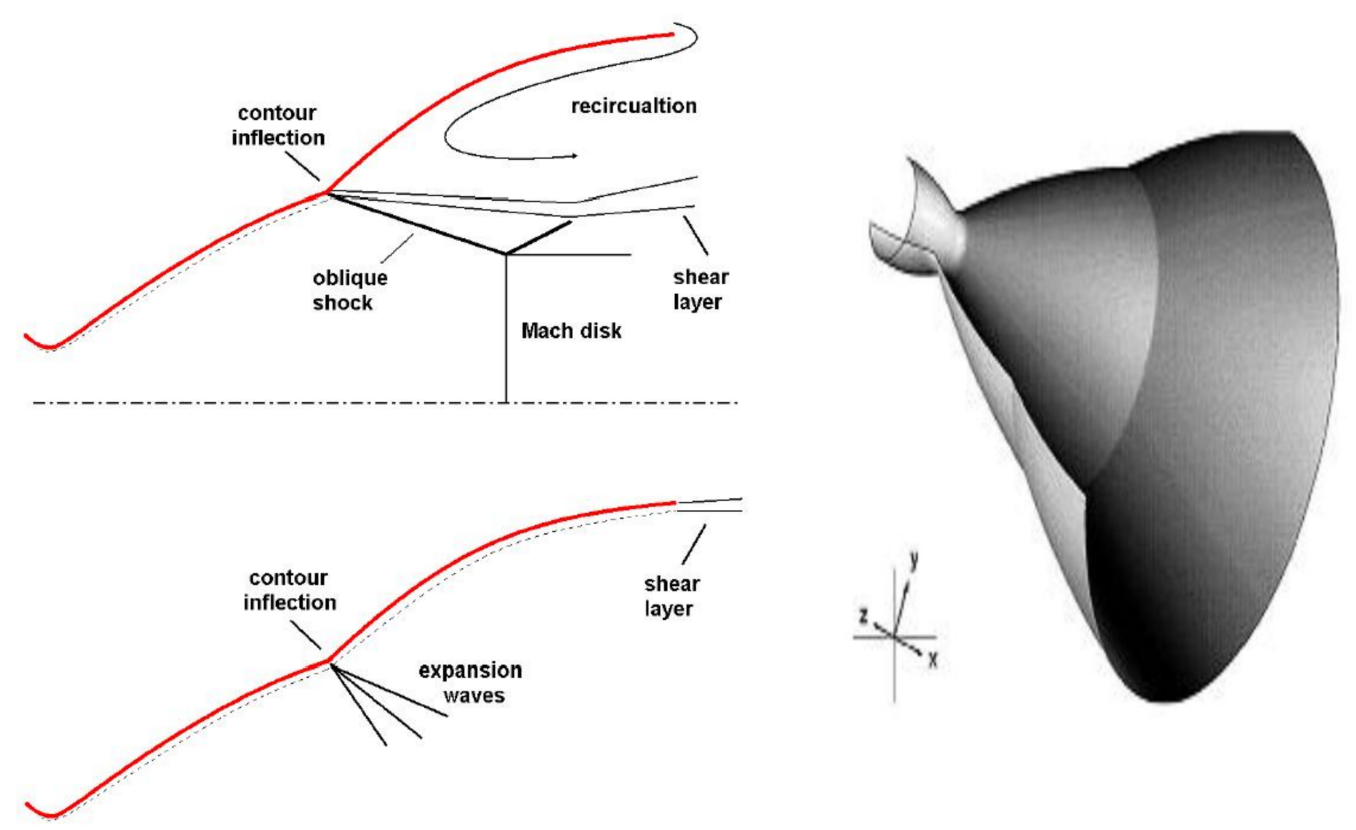

Figure 10. (left) Operational mode of a dual bell nozzle: separated under sea level mode (top) and attached in the extension under high altitude mode (bottom), (right) the dual bell nozzle in three dimensions (3-D).

\section{CFD Analysis of Ramjet Nozzles}

\subsection{CFD Setup}

For a fair comparison of the ramjet nozzle geometrical configurations analysed previously, thrust production was the primary criterion. Thrust values were obtained using Equation 4, and comparisons were conducted based on the pressure thrust $\left(\left(\mathrm{p}_{9}-\mathrm{p}_{0}\right) \mathrm{A}_{9}\right)$ and momentum thrust $\left(\left(\mathrm{m}_{9} \mathrm{~V}_{9}-\mathrm{m}_{0} \mathrm{~V}_{0}\right)\right)$ generated:

$$
F=\mathrm{m}_{9} \mathrm{~V}_{9}-\mathrm{m}_{0} \mathrm{~V}_{0}+\left(\mathrm{p}_{9}-\mathrm{p}_{0}\right) \mathrm{A}_{9}
$$

where thrust value $S$ was obtained in N. From previously available data by one of the authors [5], the design point was set for a mass flow rate of $m_{9}=m_{0}=560 \mathrm{~kg} \cdot \mathrm{s}^{-1}$ at an altitude of $50,000 \mathrm{ft}$ and at Mach 2.5. The parametric optimisation study was conducted using ANSYS Fluent in ANSYS Workbench 17, for the three nozzle types, and consisted of a data mining approach through a parametric analysis where a combination of multiple geometrical variables, i.e., length of the nozzle, curvature of the nozzle, and the height of the nozzle exit, were studied and the performance parameters compared to extract the best possible result for gross thrust production. This study focussed only on geometric constraints; details of the combustion system and flow conditions upstream of the nozzles are detailed in [5]. The secondary objective of the study was to test the effectiveness of the altitude compensating nozzle. For the purpose of simplifying the problem, a cold-flow study was employed where the extreme conditions at the nozzle inlet replicated the combustion exit conditions. A RANS (Reynolds averaged) approach to the expansion modelling was adopted due to the relatively steady state conditions and reduced computational expense [13]. The CFD study included a Weighted Area average function which noted the average pressure and velocity over the radius of the nozzle exit. The velocity and pressure results were utilised to obtain the thrust performance. Hence the performance for the best nozzle was weighed against equivalent geometrical variables, across the range of geometries modelled. 


\subsubsection{Geometry}

The geometry was constructed utilising Ansys Workbench 17. For the purpose of computational efficiency, only half of the geometry was constructed as the ramjet C-D nozzle is symmetrical. The geometry created is presented in Figure 11. Additionally, a "face split" function was featured in the geometry for further improvement of the meshing.

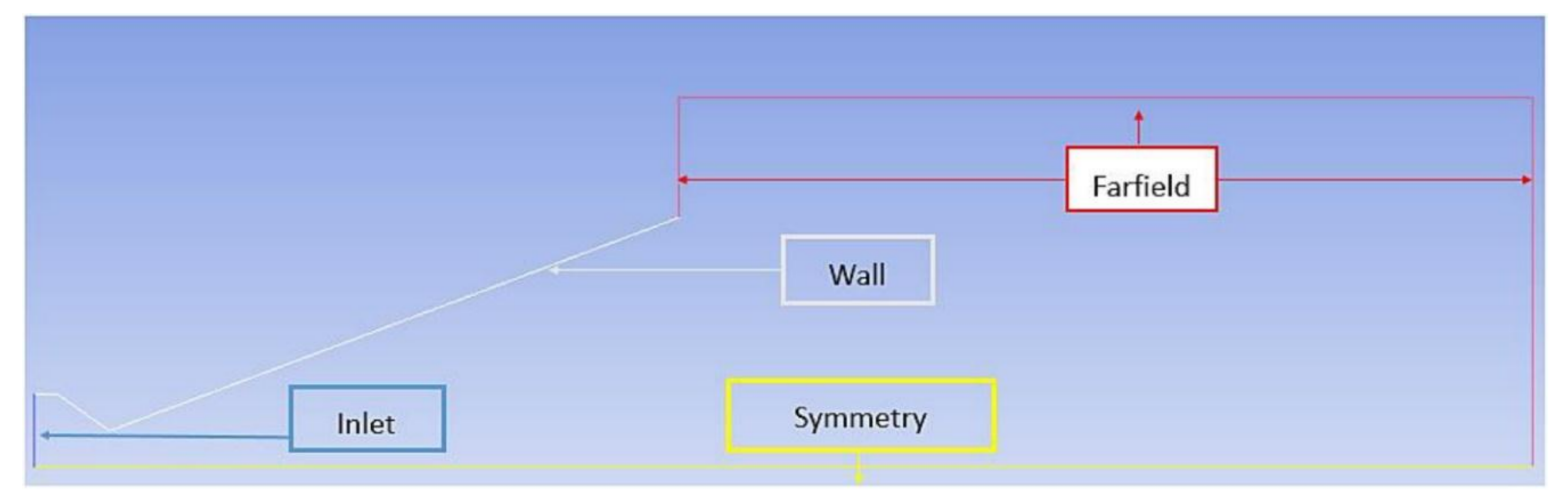

Figure 11. Dual bell nozzle geometry.

\subsubsection{Meshing}

The nozzle area was finely meshed and face-mapping was utilized, which provides an evenly spread out high-quality mesh in quadrilateral form [14]. Then, edge sizing of $0.01 \mathrm{~m}$ was added to the inner walls of the nozzle and parallel symmetry wall at the bottom to evenly spread out the number of elements throughout the nozzle horizontally.

\subsubsection{Physical Model}

The Density-Based Numerical Solver (DBNS) was selected as supersonic conditions at the nozzle exit lead to changes in density as it passes through expansion fans and shocks [15]. The solver option is suitable for sharp shock structures, which were analysed later in the study. The "K-Omega" turbulence model, a two-equation model, was selected as this model is suitable for transonic/supersonic flow and aerodynamic operations with accurate solving capabilities. The working fluid selected was ideal gas.

\subsubsection{Boundary Conditions}

The nozzle inlet boundary condition was selected as the "pressure inlet". The temperature of the combustion chamber was changed to $2000 \mathrm{~K}$ and the static pressure to $1 \times 10^{6}$ pa [16], with the pressure differential between the inlet and the ambient pressure driving the fluid forward.

The top, right, and left faces were set as the "pressure field" with a designated Mach number of 2.5 , a pressure of $11.1 \mathrm{kPA}$, and a temperature of $216 \mathrm{~K}$ to replicate the flight conditions at $50,000 \mathrm{ft}$. The walls of the nozzle were set as the "wall" with no slip condition [17]. Boundary conditions are presented in Table 2.

Table 2. Boundary conditions.

\begin{tabular}{cccc}
\hline Boundary & Pressure (Pa) & Temperature (K) & Mach Number \\
\hline Inlet (Black) & $1 \times 10^{6}$ & 2000 & N/A \\
Fairfield (Red) & $11.1 \times 10^{3}$ & 216 & 2.5 \\
Wall (White) & & N/A & \\
Symmetry (Yellow) & & N/A & \\
\hline
\end{tabular}




\subsubsection{Solution Methods and Criteria}

An implicit solution method was selected, which takes into account viscosity while acquiring solutions for the numerical problem at hand. For the Second-Order Upwind Scheme selected, the instance of numerical diffusion was greatly reduced as it assumes a multi-dimensional linear construction approach to predict face values, resulting in more accurate results than the First-Order Scheme. Thus, the Second-Order Upwind Method of interpolation was selected in Fluent.

The solver monitors in Fluent were set to absolute criteria of 1e-06 for all residuals. Doing so helped the solution to achieve a high order of accuracy. The iterative process was allowed to run until very limited change of the residual values was observed, indicating that the convergence criteria were met (Figure 12). The solution required highly accurate iterations to account for the grid network composed of a high number of elements, surpassing 120,000 for each setup.

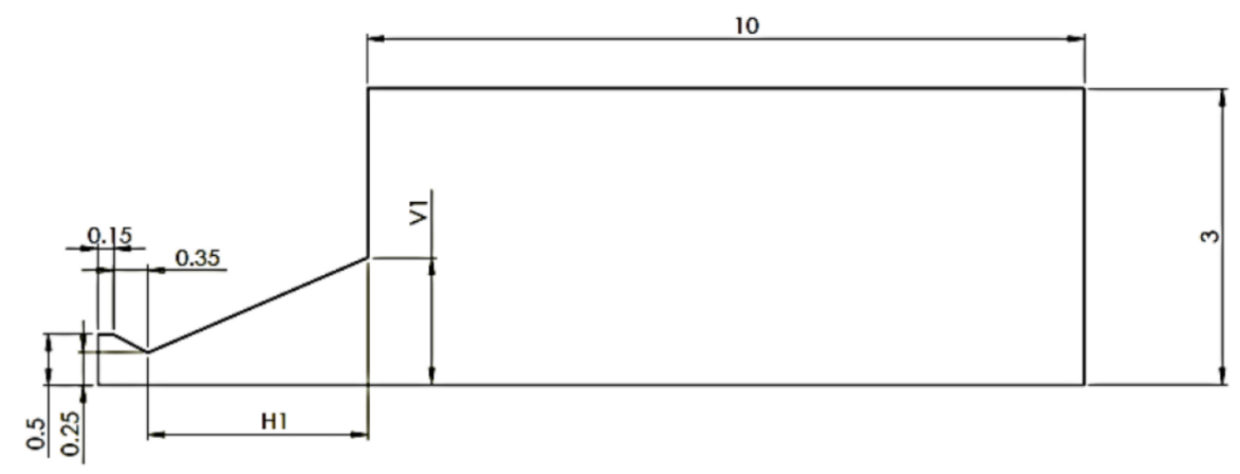

Figure 12. Conical nozzle geometry.

\subsubsection{Parametric Study}

The parametric study of the nozzle was comprised of the geometric parameters to be optimised for maximum thrust. This CFD study encompassed all the elements mentioned in the previous sections and performed the tests in an iterative manner on combinations of different geometrical variables to ensure maximisation of the performance criteria discussed previously. The method also provided insight on the comparison between the three geometries and the geometrical aspects that render one superior to another.

The conducted simulations took into account the following assumptions for compressible flow in a ramjet nozzle [18]:

- For simplicity, the exhaust gas was assumed to be an ideal gas with specific heat ratio of $\gamma=1.4$ for simplicity.

- The gas flow was isentropic, adiabatic, and frictionless.

- The gas flow was constant (i.e., steady).

- Since the exhaust accelerates exponentially, the gas flow behaviour was compressible.

- The gas flowed in a straight line along the nozzle axis of symmetry.

The details of the geometric parameters tested in the study for all three nozzles are provided below in Tables 3-5, along with the exact geometry of each tested nozzle type illustrated in Figures 12-14. The range of values selected for the bell nozzles in particular crossed the regime from TIC to TOC to investigate the effects of shifting the internal shock and enlarging the kernel zone when moving from TIC to TOC. In addition, the nozzle pressure ratio was another output parameter that resulted from the specific geometric constraints used, and this is illustrated in the figures associated with the corresponding nozzle types in the sections that follow. 
Table 3. Conical nozzle dimensional range.

\begin{tabular}{ccc}
\hline Geometrical Parameters & Parameter ID & Value Range (m) \\
\hline Nozzle Horizontal Length & H1 & $3,3.55,3.8$ \\
Nozzle Exit Radius & V1 & $1.65,1.8,2$ \\
Total Combinations & & $3 \times 3=9$ \\
\hline
\end{tabular}

Table 4. Bell nozzle dimensional range.

\begin{tabular}{ccc}
\hline Geometrical Parameters & Parameter ID & Value Range (m) \\
\hline Nozzle Horizontal Length & $\mathrm{H} 1$ & $3,3.55,3.8$ \\
Nozzle Exit Radius & $\mathrm{V} 1$ & $1.65,1.8,2$ \\
Nozzle Contour Radius & $\mathrm{R} 1$ & $6,8,10$ \\
Total Combinations & & $3 \times 3 \times 3=27$ \\
\hline
\end{tabular}

Table 5. Dual nozzle dimensional range.

\begin{tabular}{ccc}
\hline Geometrical Parameters & Parameter ID & Value Range (m) \\
\hline Nozzle Horizontal Length & H1 & $3,3.55,3.8$ \\
Outer Nozzle Exit Radius & V1 & $1.65,1.8,2$ \\
Inner Nozzle Exit Radius & V2 & $0.5,0.65,0.85$ \\
Outer Nozzle Contour Radius & R1 & $6,8,10$ \\
Inner Nozzle Contour Radius & R2 & $1,2,3,4,5,5000$ \\
Total Combinations & & $3 \times 3 \times 3 \times 6 \times 6=792$ \\
\hline
\end{tabular}

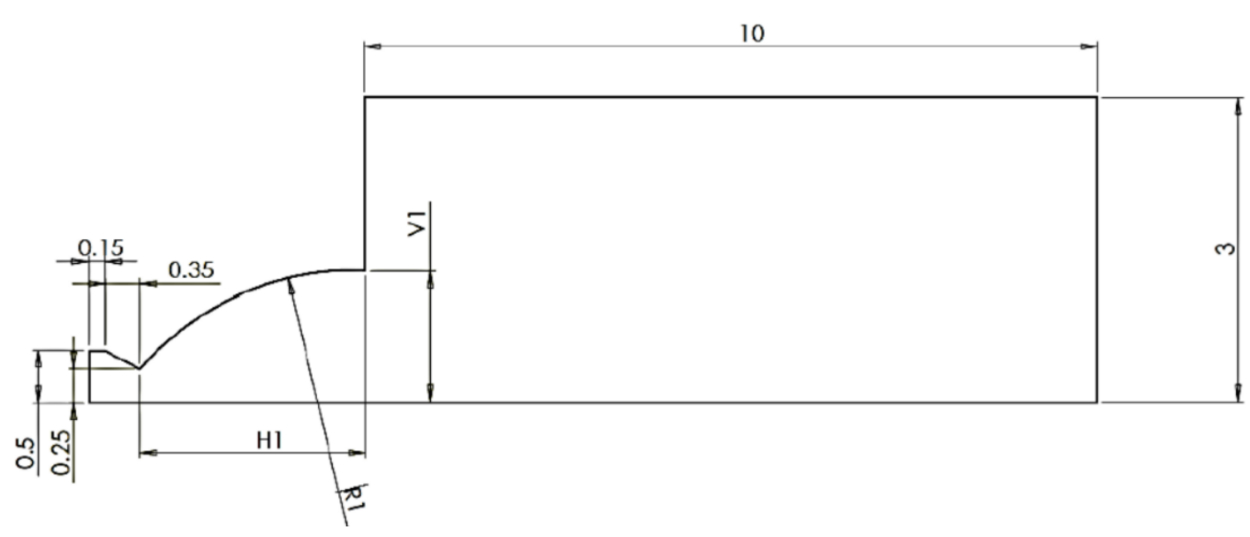

Figure 13. Bell nozzle geometry.

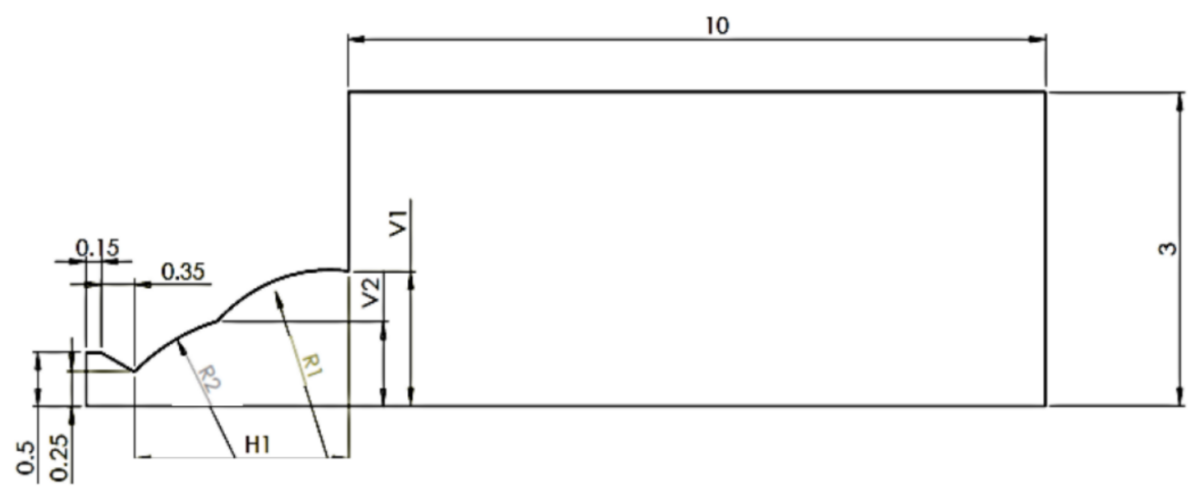

Figure 14. Dual nozzle geometry. 


\subsection{CFD Validation}

For model validation, apart from the experimental results in reference [19], the CFD study conducted in reference [12] was utilised as foundation model to be compared against the results of the present study. Table 6 provides the boundary conditions used:

Table 6. Boundary conditions for computational fluid dynamics (CFD) validation.

\begin{tabular}{ccccc}
\hline Boundary & Altitude (m) & Total Pressure (bar) & Temperature (K) & Velocity (m/s) \\
\hline Inlet & N/A & 11.5 & 2000 & N/A \\
Ambient & 10000 & 0.262 & 216 & 417 \\
\hline
\end{tabular}

As can be seen by results in Figure 15, the model was validated as the Mach contours generated for the flight altitude are almost identical. The slight variation in the shock produced in the base nozzle is caused by the internal shock produced due to the contour variation in the geometry selected.
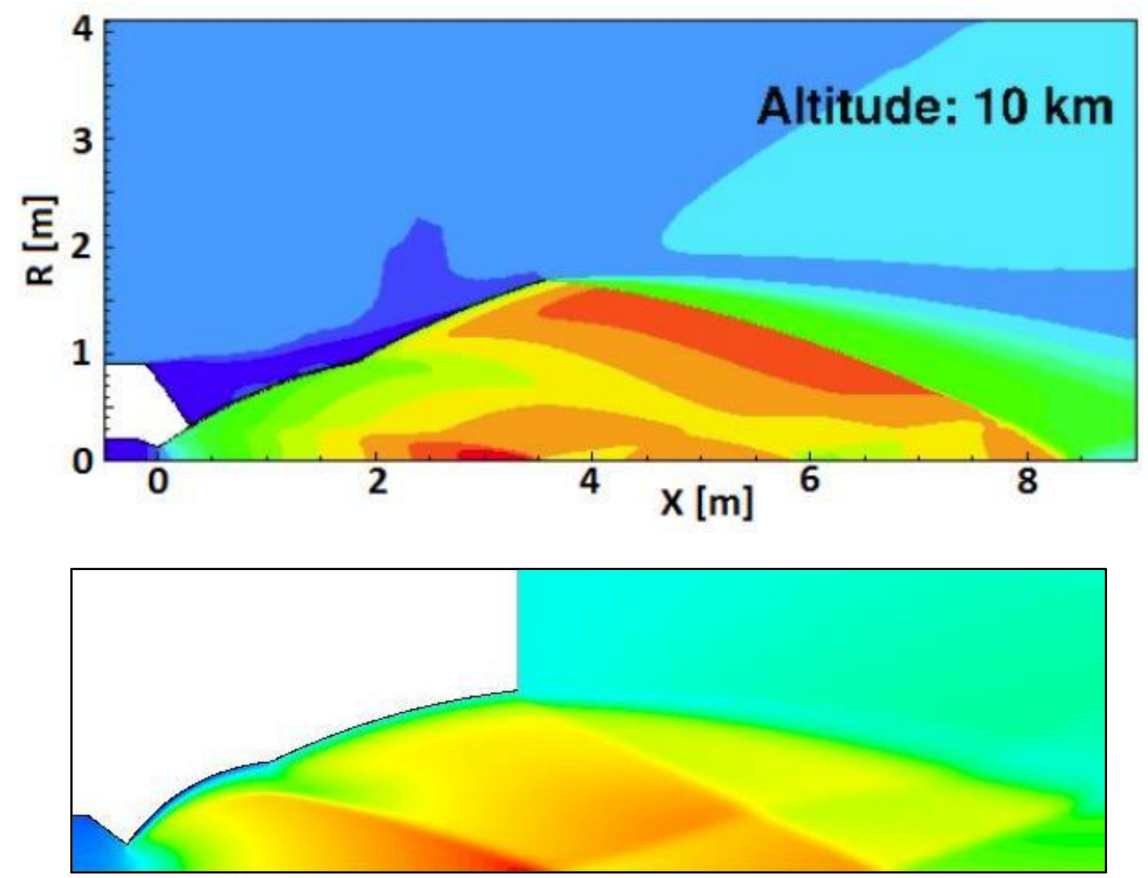

Figure 15. CFD model validations: (top) literature baseline compared against (bottom) dual bell optimised nozzle results obtained in this study.

\subsection{Conical Nozzle}

For the conical nozzle, the best thrust was obtained from the longest horizontal divergent section length of $3.8 \mathrm{~m}$, agreeing with the literature in Section 3.3.1. The pressure contour accompanied by the corresponding Mach contour in Figure 16 depicts the presence of a shock which results in an increase in pressure across the exhaust jet axis following the exit from the nozzle while the Mach decreases simultaneously. The pressure at the exit is $12 \mathrm{kPa}$ above the ambient pressure. In an ideal situation, the flow would under-expand. However, the ambient pressure causes an adverse pressure gradient on to the boundary layer at the nozzle lip which causes it to detach at the exit, inducing a shock surface line with a smaller slope towards the jet axis. The shock strengthens as it propagates towards the jet axis resulting in the reflection of the shock without producing a Mach disk. At the exhaust plume boundary, the slip line borders the jet flow, caused by the mixing of the two velocity streams. This is where the shear layer highlighted in Figure 16 is obtained. 


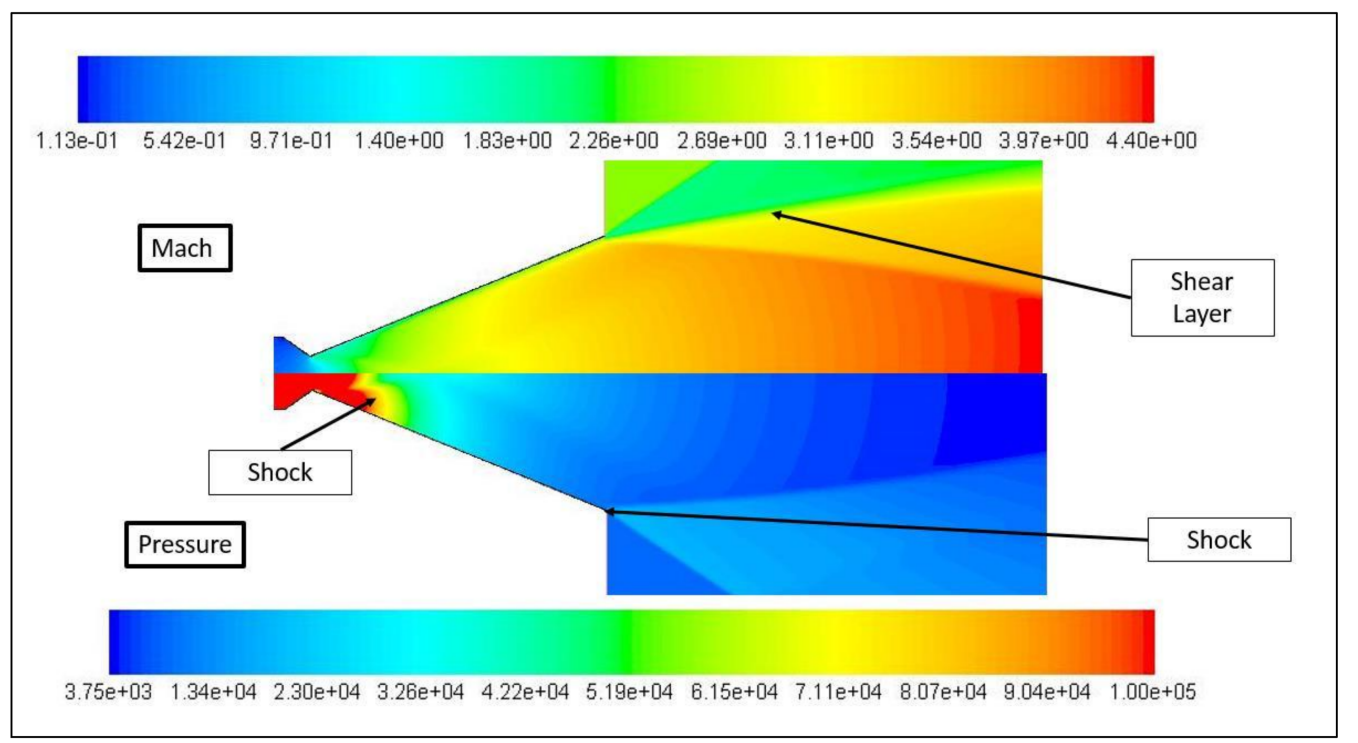

Figure 16. (Top) Pressure contour and (Bottom) Mach contour thrust $=1.47 \times 10^{6} \mathrm{~N}$, Nozzle Pressure Ratio $(N P R) \approx 100, \mathrm{H} 1=3.8, \mathrm{~V} 1=1.8$.

Thrust is mostly radial, as discussed in Section 3.3.1, where after applying the divergence factor $(\lambda)$, which was found to be 0.95 , the resultant gross axial thrust is $1.47 \times 10^{6} \mathrm{~N}$. Referring back to Figure 6 in Section 3.3.1, for a divergence angle of the best conical nozzle, i.e., $25^{\circ}$, there is a likelihood of separation for the nozzle designed. Divergence loss results in poor nozzle performance at lower altitudes resulting from flow separation. The divergence factor also accounts for the formation of separation shock in the given case. Figure 17 depicts the decrease in pressure along with an increase in Mach number along the jet axis, which validates the CFD contour maps in Figure 16. The drop in Mach at the 1.61 horizontal position is caused by the shock emanating from the sharp turn of the flow as it passes through the nozzle.

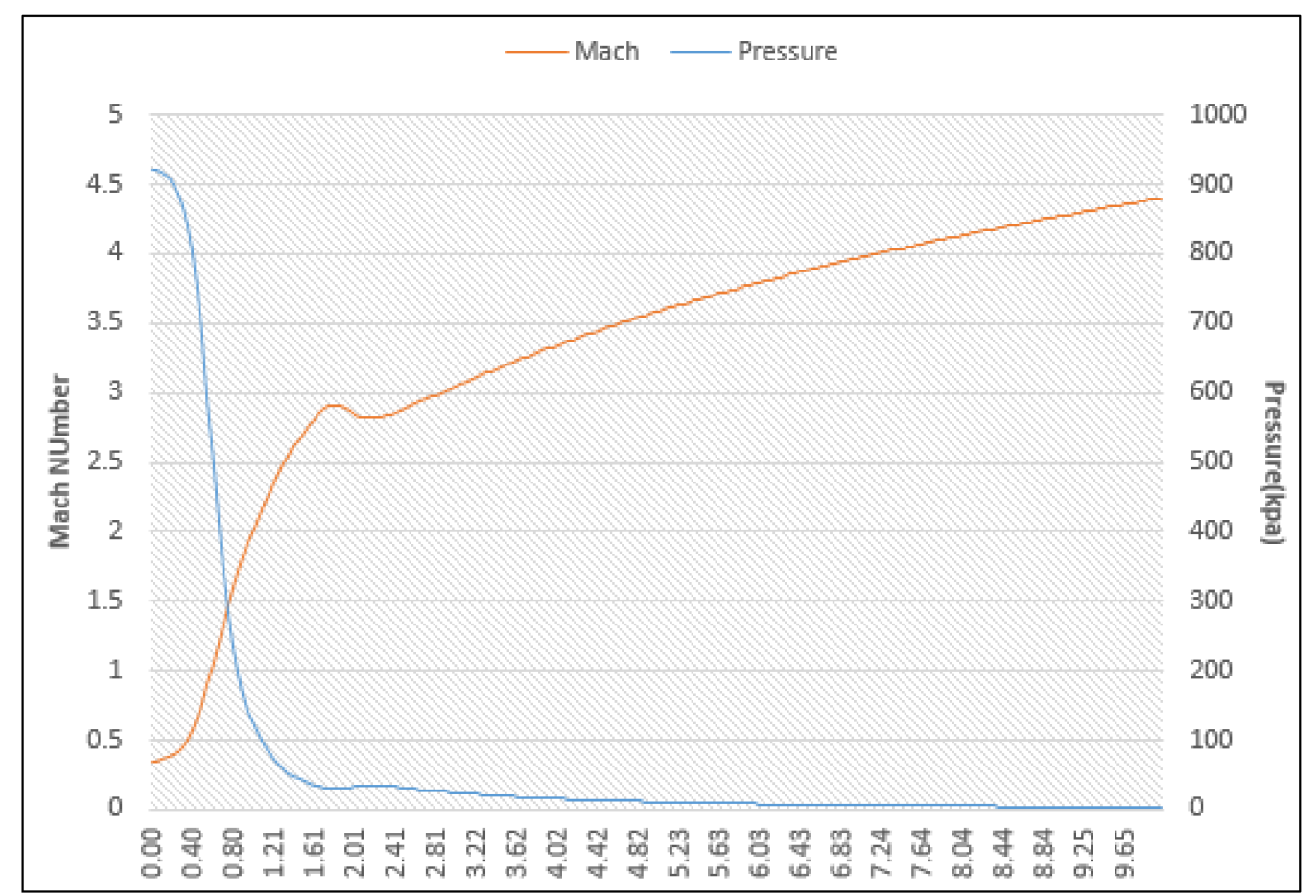

Figure 17. Mach and pressure plots against nozzle position. 


\subsection{Bell Nozzle}

Contrary to conical nozzle, the bell nozzle optimises the performance by producing a higher effective axial thrust with a lower nozzle length (H1), in this case of $3.55 \mathrm{~m}$. The expansion fan is readily visible in Figure 18, which accelerates the flow to comparatively higher Mach numbers. In addition, the thrust is far more axial as compared to the radial thrust produced by the conical nozzle previously. Additionally, internal shocks originating from the sharp corner of the throat forms a region of compression that decelerates the flow in the near wall region. Nonetheless, the contoured bell nozzle produces a relatively high exit velocity, as presented in Figure 18.

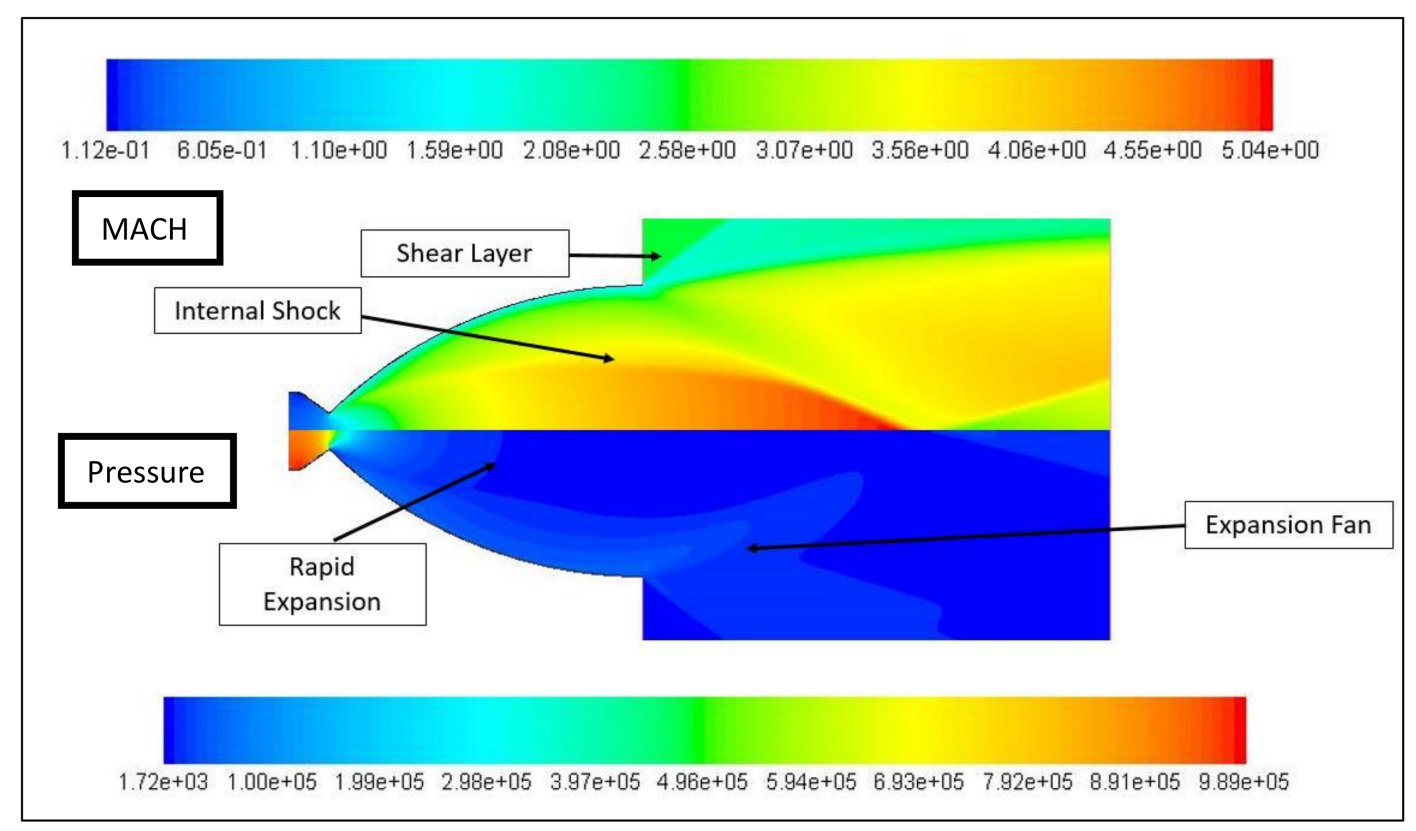

Figure 18. (Top) Pressure contour and (Bottom) Mach contour thrust $=1.58 \times 106 \mathrm{~N}, N P R \approx 90$, $\mathrm{H} 1=3.55, \mathrm{~V} 1=2, \mathrm{R} 1=6$.

Despite having a smaller exit radius and lower nozzle length, the nozzle is capable of producing a higher gross thrust value of $1.61 \times 10^{6} \mathrm{~N}$ prior to the divergence calculation, as mentioned in Section 3.3.2. The divergence factor $(\lambda)$ obtained was 0.98 , generating a resultant axial thrust of $1.58 \times 10^{6} \mathrm{~N}$ while resulting in correspondingly a lighter overall nozzle mass.

The internal shock emanating from the nozzle contour can be confirmed by utilising the vector contour plots (Figure 19) as the flow can be seen turning inwards towards the nozzle axis as the jet exits the nozzle. Similarly, the compression waves arising from the nozzle lip combine into a surface shock which changes the direction and magnitude of the flow from the radial gradient zone. The two shock waves interact and merge into a stronger shock capable of slowing down the high velocity fluid flowing through the kernel region and redirecting the flow towards the nozzle axis. As depicted in Figure 20 (right), the pressure increases considerably along the y-direction away from the nozzle axis, in agreement with the literature discussed in Section 3.3.2.

In the subsonic region, in order to efficiently shorten the effective nozzle length, the curvature radius for the contour is small but still delivers a flow over the throat. Axially downstream of the throat, where the flow is sonic, the high slope gradient allows considerable expansion. The internal shock originates from the sharp corner of the throat which distinguishes the kernel from the radial gradient zone. The nozzle selected has a design characteristic similar to that of thrust-optimised contour (TOC) nozzles, with larger curve inflection, and is capable of delivering high expansion ratios along the nozzle length [19], validating the nozzle design point. In Figures 19 and 20 the region below the shock is characterised by high momentum kernel flow which allows a higher amount of flow to 
be expanded and fully accelerated at the exit. The radial gradient region above the internal shock presents a gradual degradation of axial velocity towards the wall boundary.

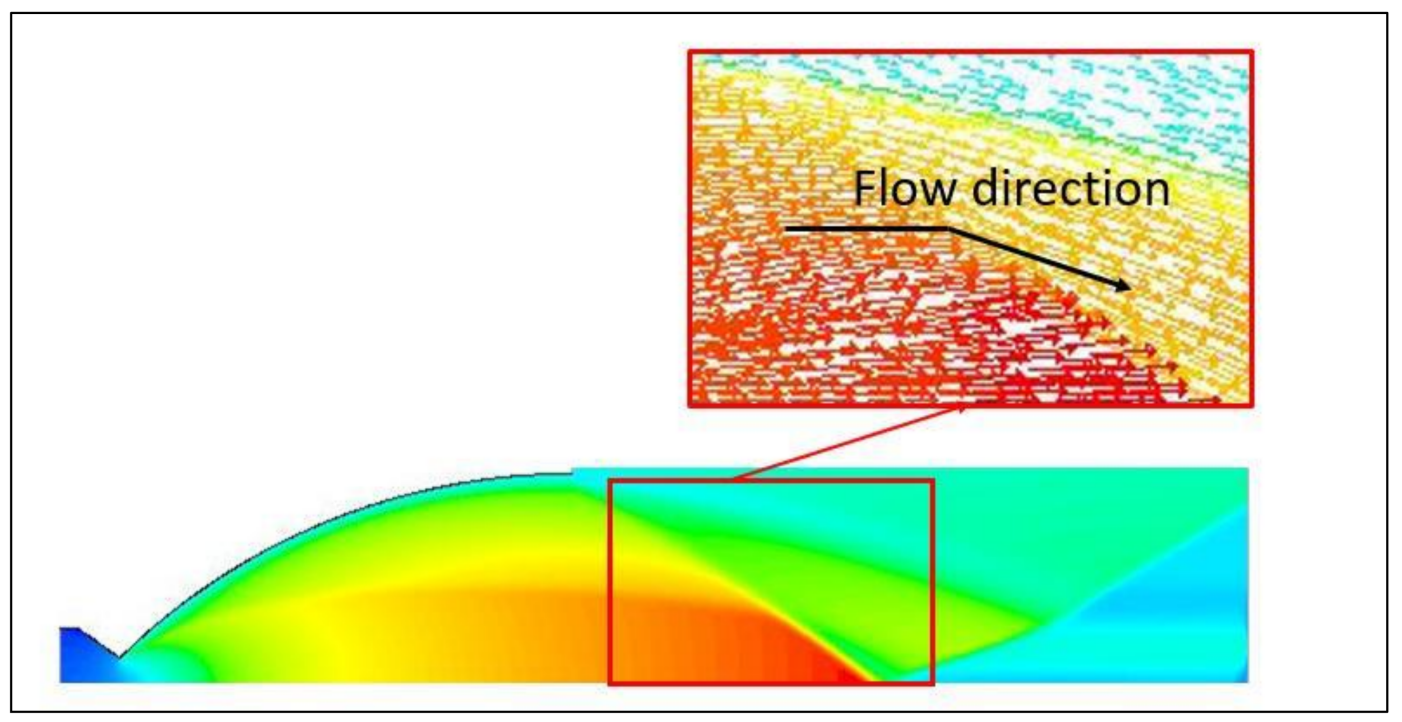

Figure 19. Evidence of shock utilising velocity vector contours.

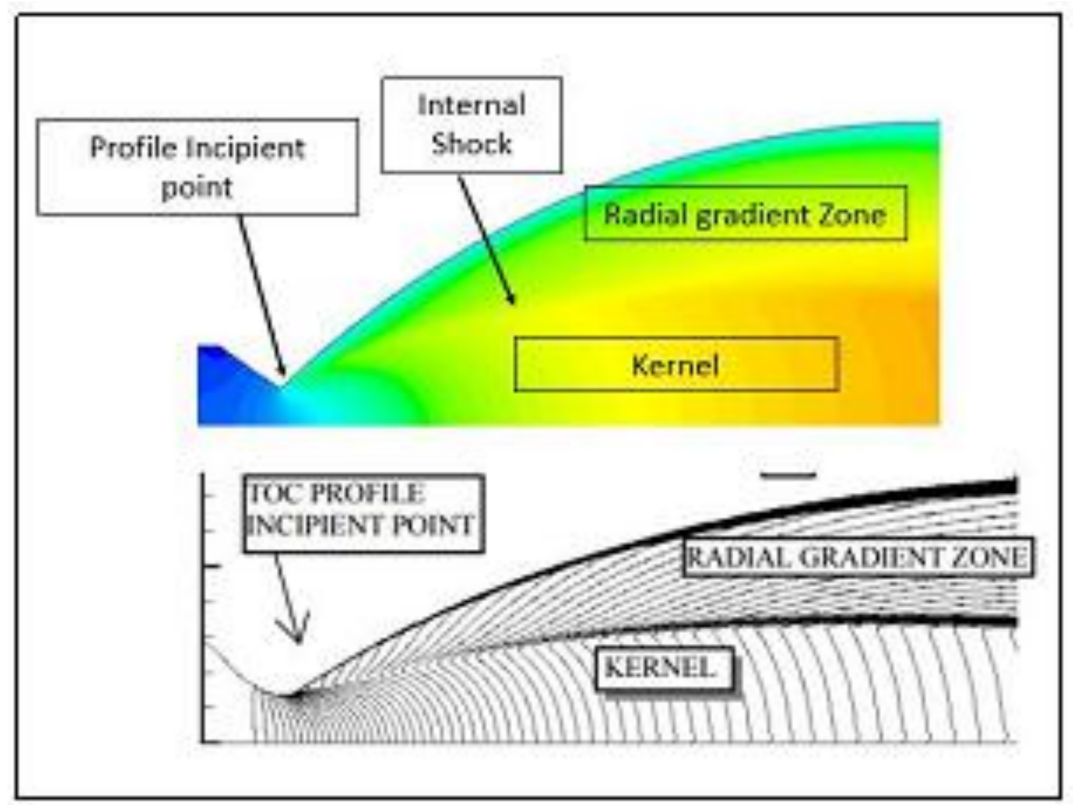

Figure 20. CFD result compared to a TOC nozzle flow pattern.

Pressure and Mach plots confirm the presence of oblique shocks where the static pressure increases while the gas decelerates dramatically downstream of the inlet. Comparison between the two nozzles of same dimensions (Figures 21 and 22) in terms of nozzle length and height indicate a higher Mach at the nozzle exit despite having the presence of an internal shock. This results in the bell nozzle producing a higher effective axial thrust instead of radial thrust. The flow in the kernel zone for the bell nozzle drops considerably, which accelerates the flow at a higher rate than the bell nozzle, leading to a higher production of momentum thrust. The pressure expansion is depicted in Figure 23 as the exhaust gas keeps expanding further into the nozzle boundaries, whereas in the conical nozzle the pressure plateaus. 


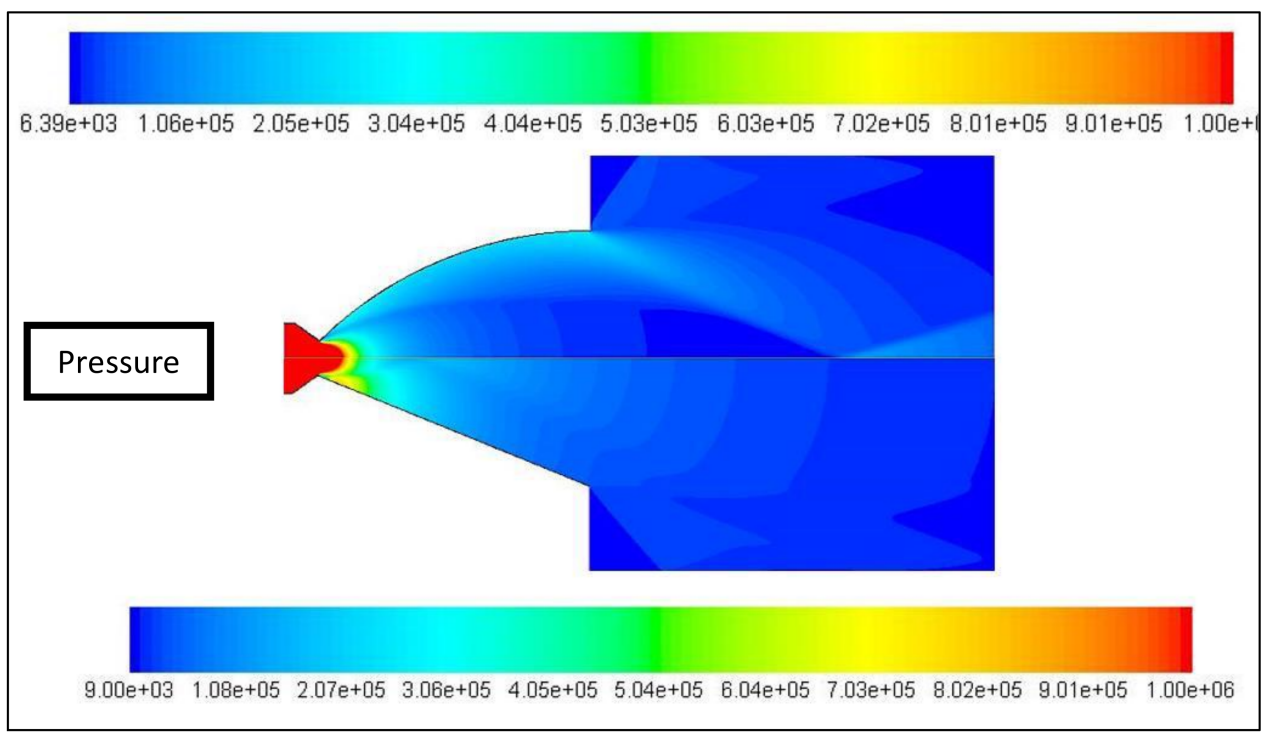

Figure 21. Pressure contour of bell nozzle (top) and conical nozzle (bottom).

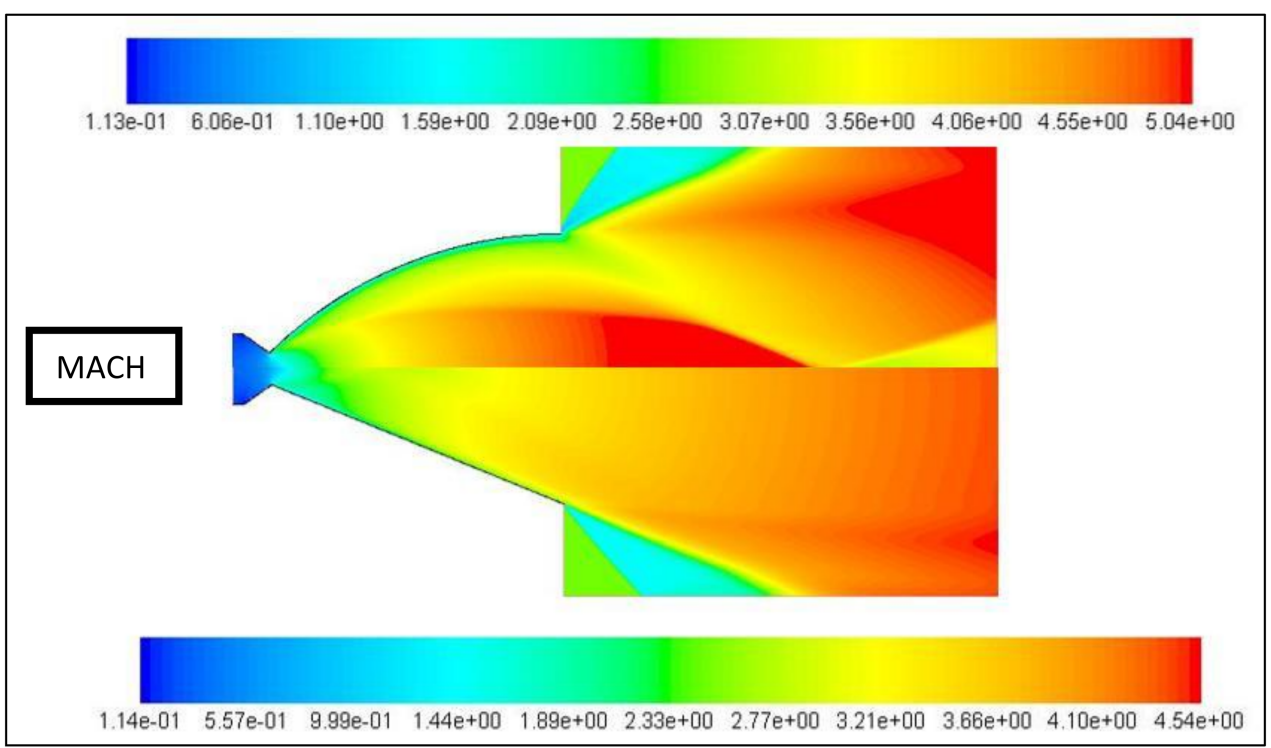

Figure 22. Mach contour for conical nozzle (top) vs. bell nozzle (bottom).

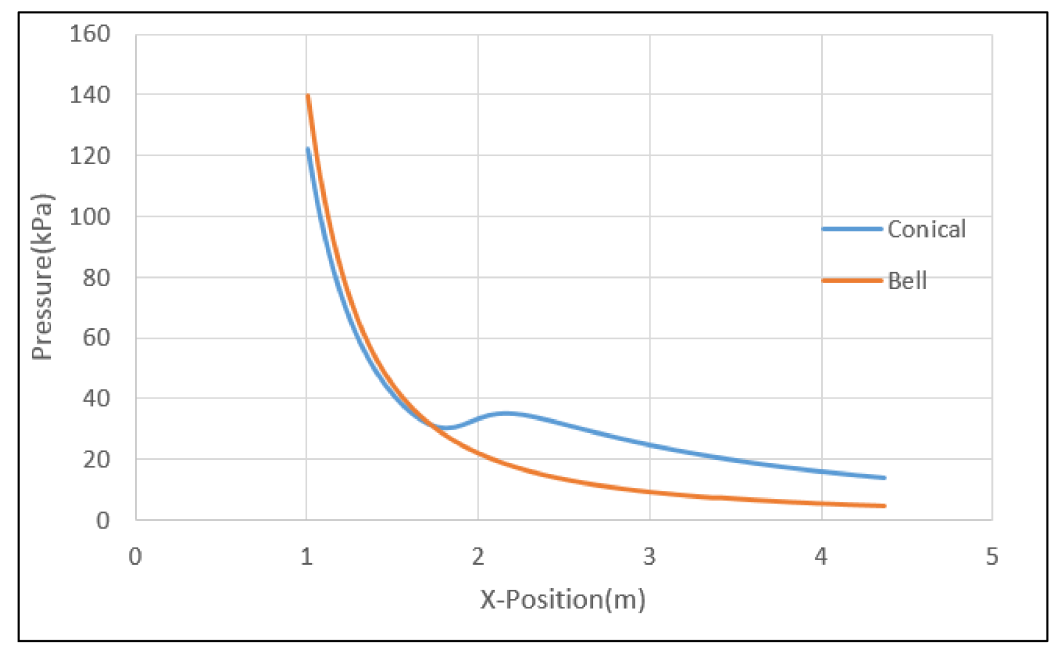

Figure 23. Pressure across the divergent section. 


\subsection{Dual Bell Nozzle}

Figure 24 depicts the performance of the dual bell nozzle at the nozzle design point. As mentioned earlier, the base nozzle suffers an oblique internal shock owing to the sharp turn in the flow as the fluid exits the nozzle throat in the nozzle. The rapid pressure drop as can be seen in the exit of the base nozzle, and the extended nozzle along the flow axis allows for acceleration along the jet axis throughout the nozzle boundaries along the kernel zone.

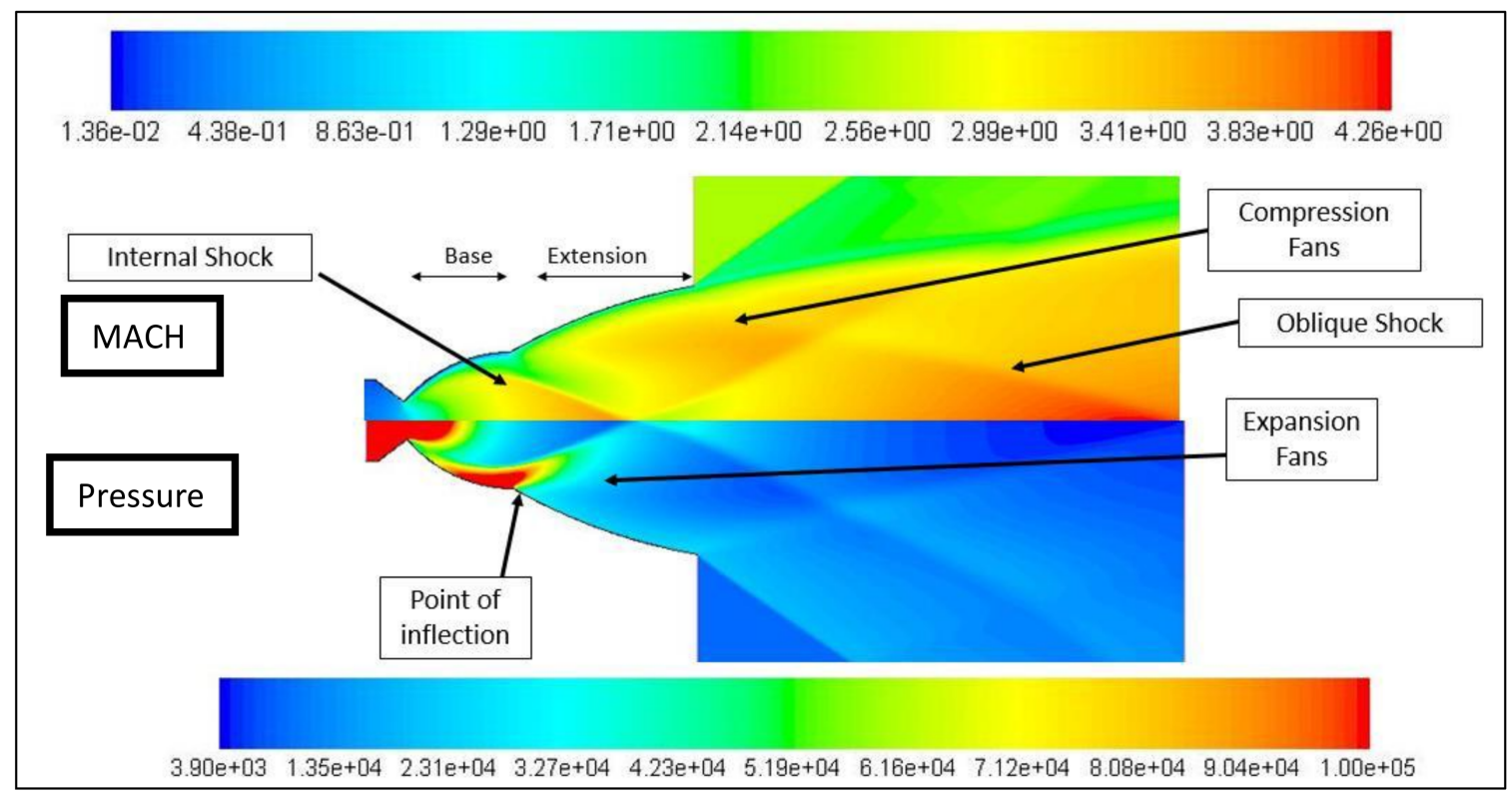

Figure 24. (Top) Pressure contour and (Bottom) Mach contour dual bell nozzle thrust $=1.89 \times 10^{6} \mathrm{~N}$, $N P R \approx 76.5, \mathrm{H} 1=2.55, \mathrm{~V} 1=1.65, \mathrm{~V} 2=0.85, \mathrm{R} 1=7, \mathrm{R} 2=1$.

The most noticeable feature of the dual bell CFD contours would be the formation of shock diamonds within the nozzle boundaries. Past the point of inflection, the flow is attached to the wall until the exit plane, where the full geometrical area ratio is utilised as discussed in Section 3.3.3.

Additionally, in the dual bell nozzle, the radial gradient region is significantly smaller with a higher overall velocity achieved throughout compared to the bell nozzle. Smaller radial gradients lead to a lower chance of separation at different altitudes, as discussed in Section 3.3. The higher velocities in the near wall region lead to the flow being less affected by the adverse pressure gradient of the outside boundary pressures. Hence, weaker shocks produced at the inflection point and the lip of the nozzle lead to efficient expansion of the exhaust throughout and beyond the nozzle. This results in a higher exit velocity, leading to considerable gain in the momentum thrust achieved by the nozzle.

The expansion fan at the lip of the nozzle exit causes the flow to expand outwards, accelerating it away from the jet axis. Compared to the other two nozzles types, the shear layer is much tamer and the jet being produced generates a much more efficient velocity and therefore a better axial thrust. At a ramjet operational altitude of 50,000 ft, the nozzle generated a gross thrust of $1.89 \times 10^{6} \mathrm{~N}$.

Figure 25 shows the pressure vs Mach plots along the axial length of this nozzle up to and beyond the nozzle throat (where the pressure drops drastically). As presented, the expansion process continues until the nozzle exit, at position $4.3 \mathrm{~m}$, where the shock induced by the nozzle lip causes the pressure to rise and the fluid interaction with the expansion fan decreases the velocity again. This leads to the formation of shock diamonds, discussed in Sections 3.2.1 and 3.2.2, that are dissipated due to the energy cascade between the two boundary flows over a course of the axial length, leading to the exhaust plume pattern. 


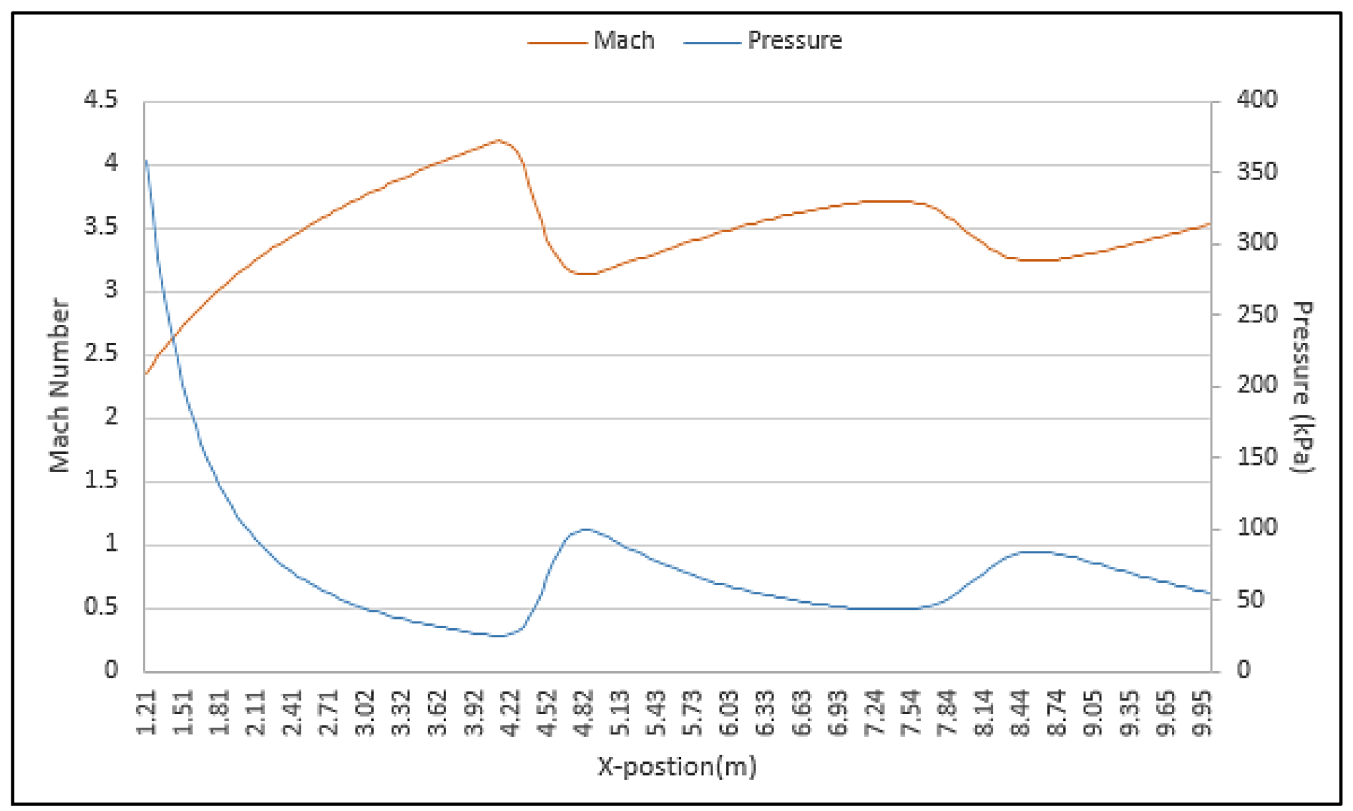

Figure 25. Pressure Mach plot across the base nozzle, extended nozzle, and beyond the exit.

\subsection{Altitude Performance of the Nozzles}

To obtain the change in altitude performance, all three nozzles were run under three different flight altitudes with the expected Mach number set according to the flight mission profile and the operational flight regime expected of the ramjet. An additional lower level of the flight at 10,000 ft was also included to scrutinise the performance of the nozzle in over-expanded conditions (see Table 7, below).

Table 7. Flight speeds at the target altitudes selected.

\begin{tabular}{cc}
\hline Altitude (m) & Mach Number \\
\hline 10000 & 1.5 \\
50000 & 2.5 \\
80000 & 4.5 \\
\hline
\end{tabular}

Figure 26 depicts the lowest tested altitude for all nozzle configurations with the best thrust performance in their respective categories. The conical nozzle performs the most efficiently, accelerating the flow through the nozzle with the highest exit velocity. Dual bell nozzle gives high overall thrust values due to the considerably higher exit velocity across the exit area plane of the nozzle since the radial gradient zone is substantially smaller. The bell nozzles are only efficient for one design point altitude, rendering them progressively less efficient away from it [20]. This leads to the dual bell nozzle having a higher effective expansion area while producing a higher axial thrust. In addition, the smaller pressure gradients at the radial zone of the dual bell nozzle reduce the likelihood of separation before the nozzle exit.

Figure 27 depicts the nozzle performance at $50,000 \mathrm{ft}$, which is the lower end of the ramjet flight regime. The bell nozzle at this stage performs considerably better than the other two nozzles with minimal radial thrust and higher exit velocity across the exit plane due to a series of expansion fans that form from the point of inflection, accelerating the flow evenly throughout nozzle plane. The conical nozzle, on the other hand, shows signs of over-expansion. The dual bell nozzle performs well, exhibiting significant flow acceleration at the kernel zone and high velocity at the nozzle exit. 


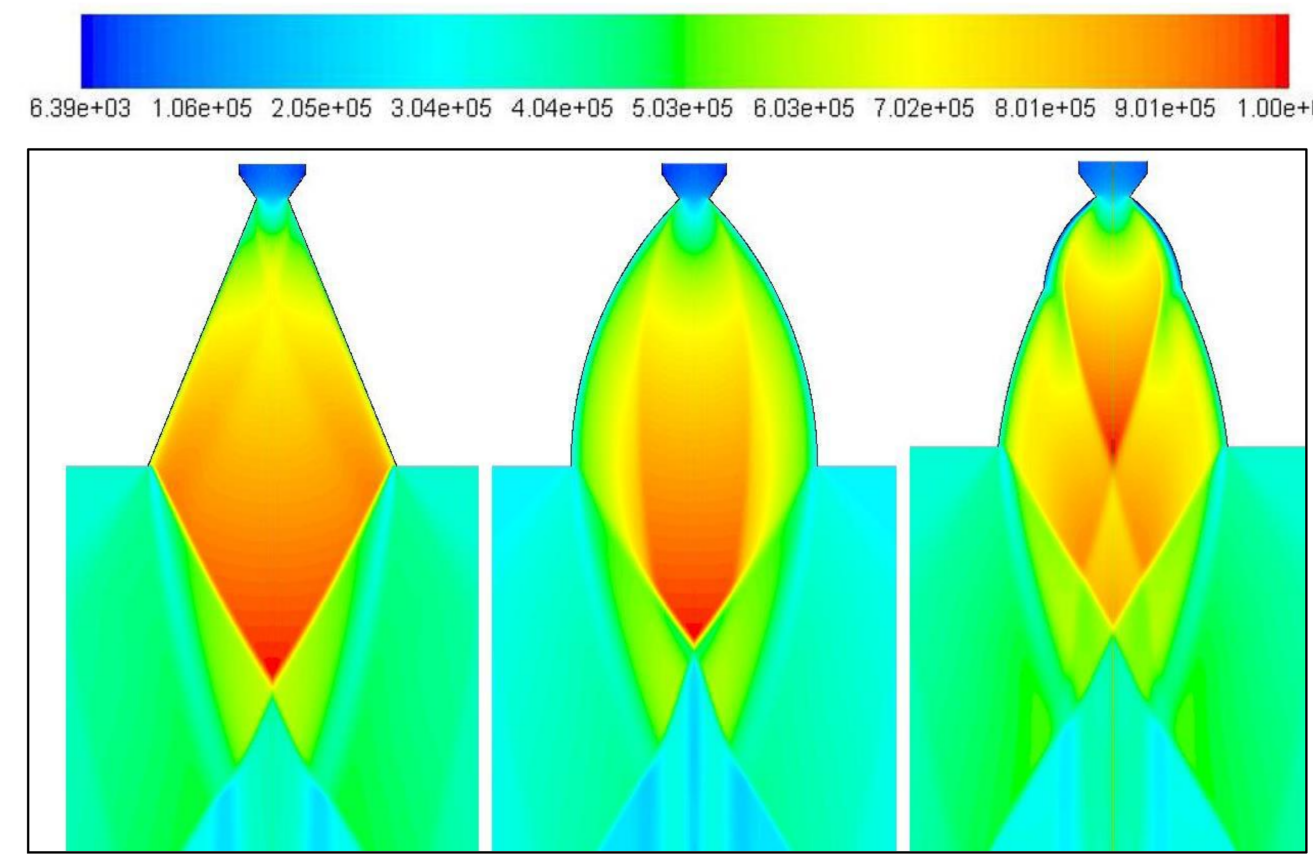

Figure 26. Pressure contours for conical, bell nozzle, and dual bell ozzle at 10,000 ft and Mach 1.5.
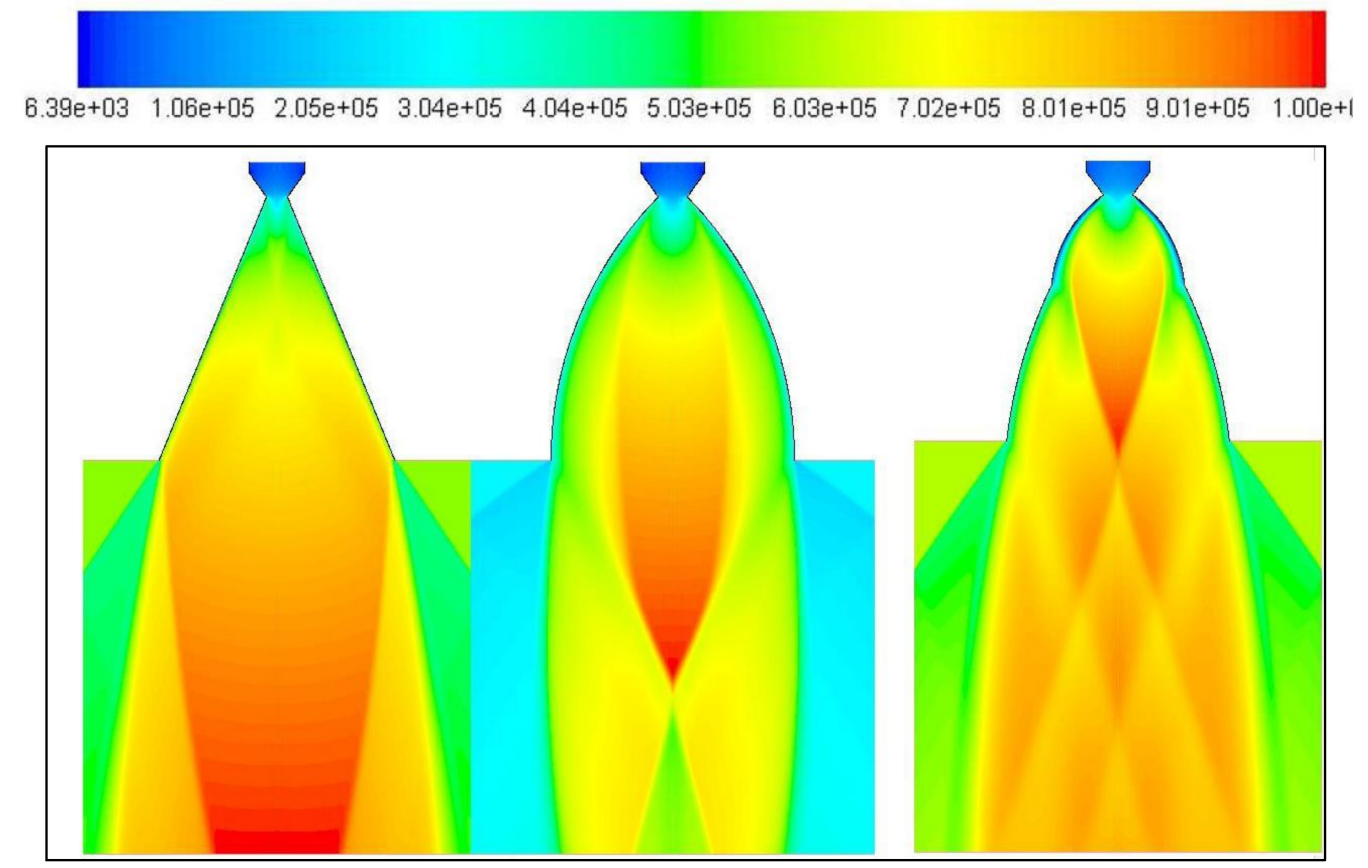

Figure 27. Pressure contours for conical, bell nozzle, and dual bell nozzle at 50,000 ft and Mach 2.5.

Figure 28, below, presents the higher end of the flight altitudes investigated. It is evident that the conical and bell nozzle performance is poor due to the comparatively higher degree of over-expansion present. Since energy released after the gas has exited the nozzle cannot be harnessed and converted into thrust, a higher degree of over-expansion leads to poor performance with inefficient utilisation of the energy stored in the combusted exhaust. The dual bell nozzle produces a much more streamlined jet with slight over-expansion characterised by the smallest shear layer thickness. 


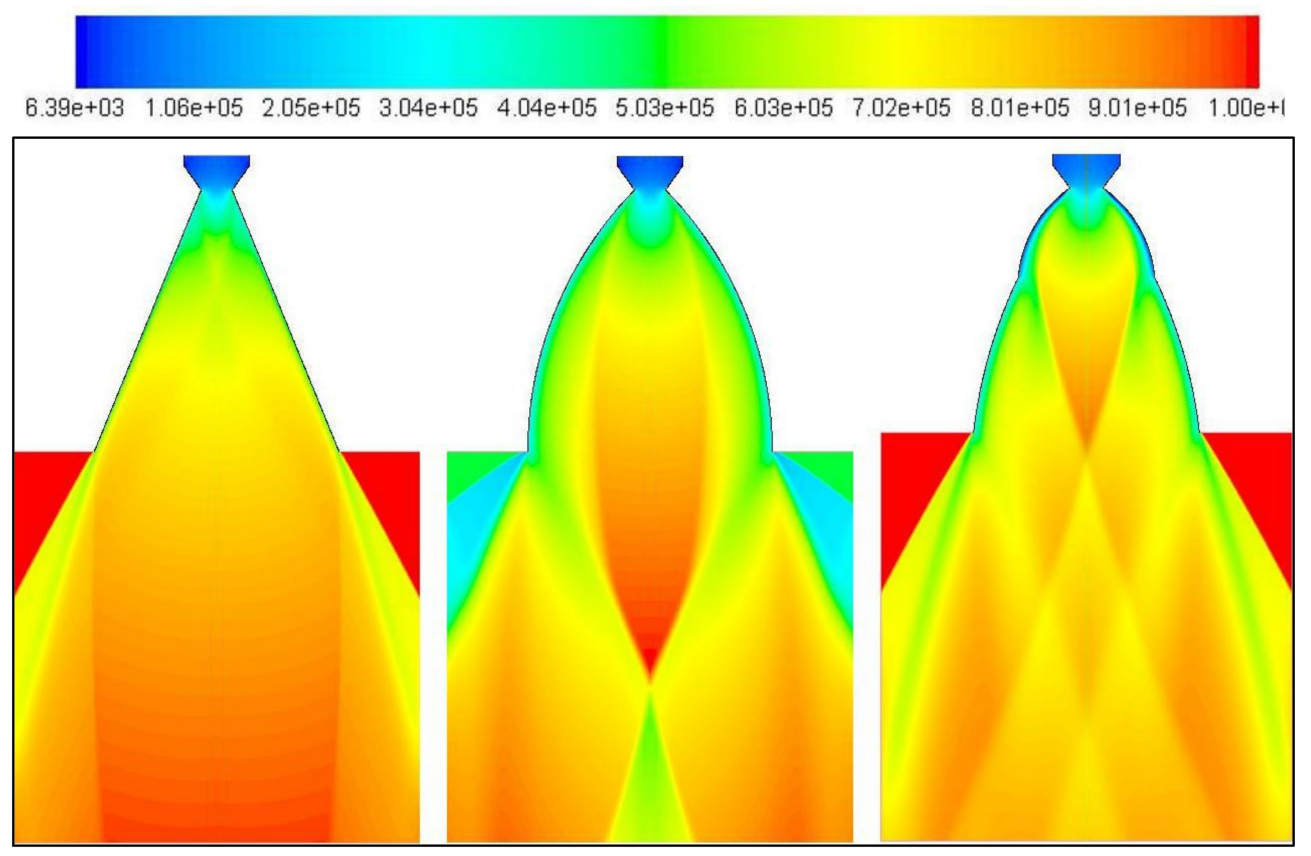

Figure 28. Pressure contours for conical, bell nozzle, and dual bell nozzle at 80,000 ft and Mach 4.5.

The final geometries considered for application in a combined cycle turboramjet-scramjet configuration are summarised in Table 8, with the dual bell nozzle being proposed as the most appropriate shape for implementation and engine integration.

Table 8. Principal dimensions and final thrust levels achieved by the three nozzles investigated.

\begin{tabular}{cc}
\hline \multicolumn{2}{c}{ Conical Nozzle } \\
\hline H1 & $3.55 \mathrm{~m}$ \\
V1 & $2 \mathrm{~m}$ \\
Gross Thrust & $1.47 \times 10^{6} \mathrm{~N}$ \\
\hline \multicolumn{2}{c}{ Bell Nozzle } \\
\hline H1 & $3.55 \mathrm{~m}$ \\
V1 & $2 \mathrm{~m}$ \\
R1 & $6 \mathrm{~m}$ \\
Gross Thrust & $1.58 \times 10^{6} \mathrm{~N}$ \\
\hline Dual Bell Nozzle \\
\hline H1 & $3.55 \mathrm{~m}$ \\
V1 & $1.65 \mathrm{~m}$ \\
V2 & $0.85 \mathrm{~m}$ \\
R1 & $7 \mathrm{~m}$ \\
R2 & $1 \mathrm{~m}$ \\
Gross Thrust & $1.89 \times 10^{6} \mathrm{~N}$ \\
\hline
\end{tabular}

The three-dimensional (3-D) shape of the nozzle put forward with the dimensions and geometry provided in Table 8 is presented in Figure 29, below. 


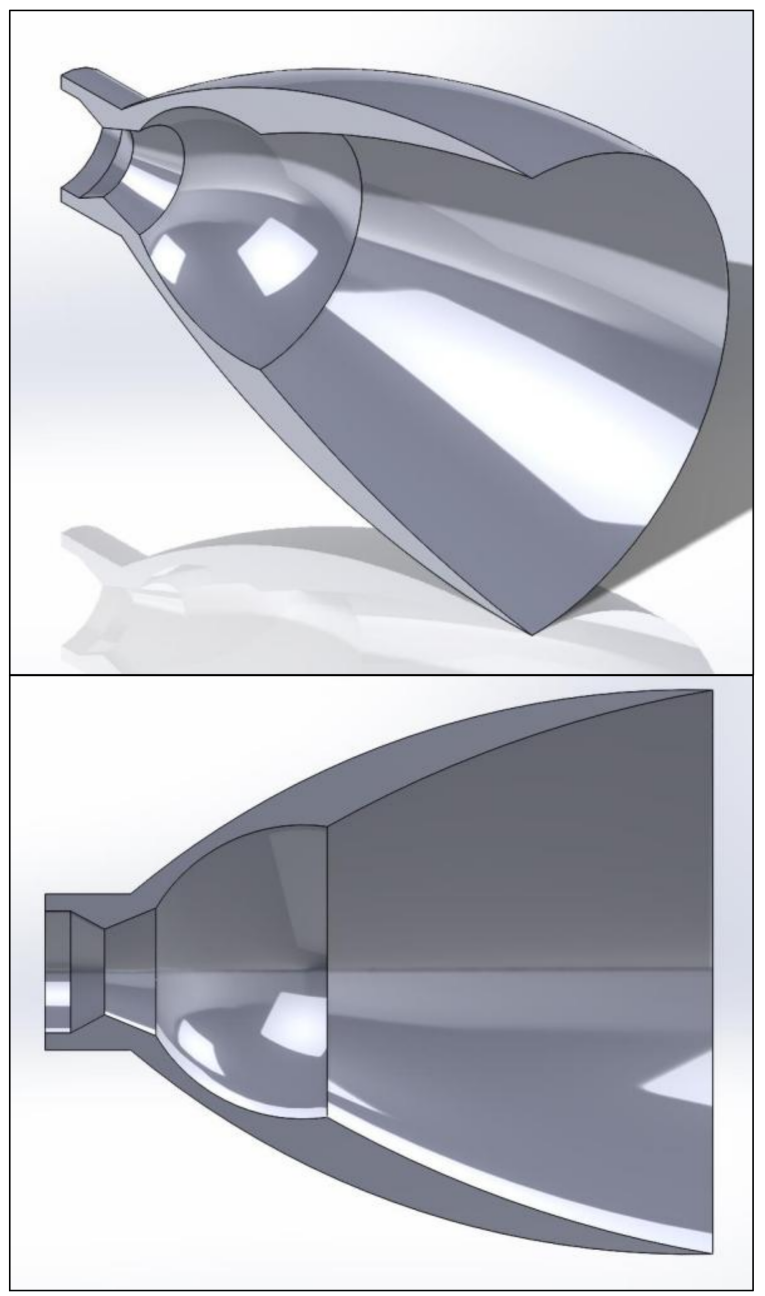

Figure 29. Cross-sectional view of nozzle: isometric (top) and side (bottom) view

\section{Conclusions}

A nozzle performance study was conducted with three principal nozzles considered for ease-of-implementation for a hypersonic combined cycle propulsion system consisting of a turbo-jet, ramjet, and scramjet configuration for flight speeds up to Mach 8.

The nozzles considered were of the conical, bell, and dual bell types and were computationally tested under various flight conditions of $10000 \mathrm{ft}$ at Mach 1.5, 50,000 ft at Mach 2.5, and 80,000 ft at 4 Mach (with the design point being the $50,000 \mathrm{ft} / \mathrm{M} 2.5$ case), which were the expected flight speed/altitude combinations of their expected operation. All three optimised nozzles displayed proficient performance throughout the ramjet operational flight profile.

The conical nozzle displayed good thrust performance at sea level. However, with altitude gain, the divergence factor caused it to lose thrust significantly as the exhaust gas began expanding beyond the nozzle exit contours radially.

The bell nozzle performed well at the design point altitude of 50,000 ft. However, it failed to perform at lower or higher altitudes with lower exit velocities and lower axial thrust being produced. Additionally, at the highest altitude, the under-expansion led to poor pressure thrust production in the nozzle.

The design point altitude for the dual bell nozzle was at 50,000 ft, where the other two nozzles under-performed. The nozzle performance was minimally compromised as the numerical study depicted high thrust values at various altitudes without suffering major consequences from over- or 
under-expansion at different flight altitudes. As a result, the dual bell nozzle was put forward for engine integration in the combined cycle hypersonic propulsion architecture.

Author Contributions: Raman Baidya and Maxim Cooper were the research students that conducted the detailed study and wrote the first draft of this paper. Apostolos Pesyridis conceived of the project, created the layout of the investigations, and checked the computational outcome of the resultant modelling effort and subsequent discussion.

Conflicts of Interest: The authors declare no conflict of interest.

\section{References}

1. Lockheed Martin Corporation. Creating the Blackbird. Available online: http:/ /www.lockheedmartin.co. uk/us/100years/stories/blackbird.html (accessed on 25 March 2017).

2. Jenkins, D.R. X-15: Extending the Frontiers of Flight; NASA SP-2007-562; NASA: Washington, DC, USA, 2007.

3. Calvert, B. Flying Concorde, 1st ed.; Airlife Publishing: Shrewsbury, UK, 2002.

4. Heiser, W.; Daley, D.; Pratt, D. Hypersonic Airbreathing Propulsion; American Institute of Aeronautics and Astronautics: Washington, DC, USA, 1994; ISBN 978-1-56347-035-6.

5. Neill, S.M.; Pesyridis, A. Modeling of Supersonic Combustion Systems for Sustained Hypersonic Flight. Energies 2017, 10, 1900. [CrossRef]

6. Bragg, S. Rocket Engines, 1st ed.; George Newnes: London, UK, 1962.

7. Kirk, D.R.; Archambault, M.R. MAE 4262: Rockets and Mission Analysis; Florida Institute of Technology: Melbourne, FL, USA, 2017.

8. Ekanayake, S.; Gear, J.; Ding, Y. Flow simulation of a two dimensional rectangular supersonic convergent divergent nozzle. ANZIAM J. 2010, 51, 377. [CrossRef]

9. Aerospaceweb.org. Aerospaceweb.org|Ask Us—Shock Diamonds and Mach Disks. Available online: http:/ / www.aerospaceweb.org/question/propulsion/q0224.shtml (accessed on 11 April 2017).

10. Davis, K.; Fortner, E.; Heard, M.; McCallum, H.; Putzke, H. Experimental and computational investigation of a dual-bell nozzle. In Proceedings of the 53rd AIAA Aerospace Sciences Meeting (AIAA 2015-0377), Kissimmee, FL, USA, 5-9 January 2015.

11. Hagemann, G.; Immich, H.; Nguyen, T.; Dumnov, G. Advanced Rocket Nozzles. J. Propuls. Power 1998, 14, 620-634. [CrossRef]

12. Stark, R.; Genin, C.; Wagner, B.; Koschel, K. The Altitude Adaptive Dual Bell Nozzle. In Proceedings of the 16th International Conference on the Methods of Aerophysical Research (ICMAR 2012), Kazan, Russia, 20-26 August 2012.

13. Schomberg, K.; Olsen, J.; Doig, G. Analysis of a low-angle annular expander nozzle. Shock Vib. 2015, 2015, 1-8. [CrossRef]

14. Sharcnet.ca. Notes on Face Meshing Controls for Mapped Meshing. Available online: https://www.sharcnet. ca/Software/Ansys/17.0/en-us/help/wb_msh/msh_Notes_Map_Face.html (accessed on 17 April 2017).

15. Sasanapuri, B.; Kumar, M.; Wirogo, S. Simulation of Flow through Supersonic Cruise Nozzle: A Validation Study; Thermal \& Fluids Analysis Workshop, NASA Langley Research Center: Washington, DC, USA, 2011.

16. Hagemann, G.; Frey, M. Shock pattern in the plume of rocket nozzles: needs for design consideration. Shock Waves 2008, 17, 387-395. [CrossRef]

17. Pandey, K.; Singh, A. CFD analysis of conical nozzle for mach 3 at various angles of divergence with fluent software. Int. J. Chem. Eng. Appl. 2010, 1, 179-185. [CrossRef]

18. Venkatesh, V.; Reddy, J. Modelling and Simulation of Supersonic Nozzle Using Computational Fluid Dynamics. Int. J. Novel Res. Interdiscip. Stud. 2015, 2, 16-27.

19. Munday, D.; Gutmark, E.; Liu, J.; Kailasanath, K. Flow structure and acoustics of supersonic jets from conical convergent-divergent nozzles. Phys. Fluids 2011, 23, 116102. [CrossRef]

20. Davenas, A. Solid Rocket Propulsion Technology, 1st ed.; Pergamon Press: Oxford, UK, 1993. 